DESY 96-081

HD-THEP-96-11

hep-th/9605039

\title{
Quantum Liouville Field Theory as Solution of a Flow Equation
}

\author{
M. Reuter \\ Deutsches Elektronen-Synchrotron DESY \\ Notkestrasse 85, D-22603 Hamburg \\ C. Wetterich \\ Institut für Theoretische Physik \\ Universität Heidelberg \\ Philosophenweg 16, D-69120 Heidelberg
}

\begin{abstract}
A general framework for the Weyl invariant quantization of Liouville field theory by means of an exact renormalization group equation is proposed. This flow equation describes the scale dependence of the effective average action which has a built-in infrared cutoff. For $c<1$ it is solved approximately by a truncation of the space of action functionals. We derive the Ward identities associated to Weyl transformations in presence of the infrared cutoff. They are used to select a specific universality class for the renormalization group trajectory which is found to connect two conformal field theories with central charges $25-c$ and $26-c$, respectively.
\end{abstract}




\section{Introduction}

In spite of the fact that all its classical solutions are known for more than a century [1], a satisfactory treatment of quantum Liouville field theory is still lacking. Although a variety of approaches, including canonical quantization [2], [3], [4], [5], inverse scattering techniques [6], path integral [7], quantum group [8], [9] and topological field theory methods [10], have provided important insights into the structure of the theory, it is still not possible to calculate arbitrary $n$ point functions or to get a handle on the notoriously difficult question about the structure of its vacuum. Liouville theory is certainly a fascinating topic also in its own right, but much of the recent interest in this theory is due to its relevance to noncritical string theory [11] and to 2-dimensional quantum gravity. For $c>25$ it was also suggested to model essential aspects of 4-dimensional quantum gravity [12]. The subject was further stimulated by the comparison with alternative approaches such as quantum gravity in the light-cone gauge [13], [14] or matrix models 15 .

In this paper we shall quantize Liouville field theory within the general framework of the exact renormalization group approach [16]. This method has led to an improved qualitative understanding and conceptual clarification of many issues in renormalization theory. It has been extended in various directions and its relation with the concept of a coarse-grained free energy has been clarified [17]- 28]. More precisely, we are using a formulation in terms of the effective average action [18][26]. The average action $\Gamma_{k}$ [18 is a modification of the standard effective action $\Gamma$ with a built-in infrared cutoff $k$. I It is the effective action appropriate for fields which have been averaged over spacetime volumes of size $k^{-1}$. Stated differently, $\Gamma_{k}$ obtains from the classical action $S$ by integrating out only the field modes with momenta larger than $k$, but excluding the modes with momenta smaller than $k$. In the limit $k \rightarrow 0, \Gamma_{k}$ approaches the standard effective action, $\Gamma_{k \rightarrow 0}=\Gamma$. Here $\Gamma$ is the generating functional for the 1PI Green's functions and includes all quantum fluctuations. The dependence of $\Gamma_{k}$ on the scale $k$ is described by an exact non-

\footnotetext{
${ }^{1}$ For a review see ref. [26.
} 
perturbative flow equation [20], 21]. This functional differential equation takes the form of a renormalization group-improved one-loop equation [18], [20], where the classical inverse propagator is replaced by the full inverse propagator, i.e., the second functional derivative of $\Gamma_{k}$ with respect to the fields.

The quantization of a theory in terms of the effective average action proceeds in the following steps: One first specifies the "short-distance action" or "microscopic action" $S=\Gamma_{\Lambda}$ as the "initial value" of the average action specified at some large scale $k=\Lambda$. This specification regularizes the theory completely if the infrared cutoff is efficient enough, i.e., if the $k$-derivative of $\Gamma_{k}$ is ultraviolet finite. One may consider the limit $\Lambda \rightarrow \infty$, but this is not necessary and it is often more instructive to keep a finite "ultraviolet cutoff scale" $\Lambda$. On the other hand, the quantum theory is completely given by the effective action $\Gamma$. Quantization of a theory amounts then to the solution of the flow equation for $k \rightarrow 0$, starting with initial values at the scale $\Lambda$. This procedure is rather general, but for its practical implementation a few comments are in order.

i) While the flow equation is exact, it is usually not possible to find exact solutions of it. There exists, however, a rather attractive approximation scheme which is nonperturbative in nature and does not require a small expansion parameter. This method consists of truncating the space of all action functionals, and to project the renormalization group flow on a finite dimensional subspace. It is this technique of projected renormalization group equations which we shall apply to Liouville theory.

ii) In consequence, there are two basic ingredients in this approach. The first is the existence of an exact flow equation which can serve as a starting point for the approximations. On this level our formulation is not much different from many earlier versions of exact renormalization group equations [16], [17, since all these formulations can be related by suitable transformations. The second ingredient is the possibility of an efficient truncation. This means that the space of truncated actions must be small enough to permit a computation and large enough to contain all "relevant physics". For a judgement if the "relevant physics" is described by a given truncation, the exact formulation of the effective action and the specific 
form of the flow equation are of crucial importance. We believe that it is useful in this respect to concentrate the emphasis on the variation of an infrared cutoff since the problematic parts of the "quantization" typically involve a complicated infrared behavior. This can be understood easily in the case of massless threedimensional scalar theories which are superrenormalizable in the ultraviolet, but have a complicated infrared behavior with fixed points and critical exponents. In addition, the most important general properties of $\Gamma_{k}$ must be known in order not to omit important effects by the truncation. For this purpose, the free energy with an infrared cutoff, $\Gamma_{k}$, is a very useful quantity since one often has some experience of the general effects of quantum fluctuations and of the consequences if the small momentum fluctuations $\left(q^{2}<k^{2}\right)$ are omitted. The close analogy of our flow equation with renormalization group improved one-loop perturbation theory helps considerably in this respect.2

iii) The infrared cutoff must be quadratic in the quantum fields $\chi$ as given by an additional piece in the action

$$
\Delta_{k} S=\frac{1}{2} \int \frac{d^{d} q}{(2 \pi)^{d}} \bar{\chi}(q) \mathcal{R}_{k}\left(q, q^{\prime}\right) \chi\left(q^{\prime}\right)
$$

with $\mathcal{R}_{k}$ typically given by $\mathcal{R}_{k}=R_{k}(q)(2 \pi)^{d} \delta\left(q-q^{\prime}\right)$ and $R_{k}(q)$ a function of $q^{2}$ that should vanish identically for $k \rightarrow 0$. This results in the flow equation $\left(\partial_{t}=\partial / \partial \ln k\right)$

$$
\partial_{t} \Gamma_{k}=\frac{1}{2} \operatorname{Tr}\left\{\left(\partial_{t} \mathcal{R}_{k}\right)\left(\Gamma_{k}^{(2)}+\mathcal{R}_{k}\right)^{-1}\right\}
$$

with Tr containing a momentum integral. In four dimensions a masslike infrared cutoff $R_{k}=Z k^{2}$ is usually not sufficient to guarantee ultraviolet finiteness of the

\footnotetext{
${ }^{2}$ Note that a relatively simple form of the effective average action $\Gamma_{k}$ (which is 1PI-irreducible) typically results in a quite complicated form of the "ultraviolet cutoff action" (1PI reducible) whose evolution is described by earlier versions of exact renormalization group equations [16]. In presence of massless particles and in the momentum range $q^{2} \gg k^{2}$ a local form of $\Gamma_{k}$ corresponds to a nonlocal "ultraviolet cutoff action". This makes it very difficult to find nonperturbative truncations for the "ultraviolet cutoff action". This problem is probably the reason why most earlier attempts to solve the exact renormalization group equations have remained in a perturbative context (small coupling, small $\epsilon=4-d$, large number $N$ of components). For more complicated theories such as non-abelian gauge theories it is in addition notoriously difficult to formulate a satisfactory ultraviolet cutoff.
} 
r.h.s.. (More precisely, only the field-dependent part of $\Gamma_{k}$ is of interest.) Our proposal requires that $R_{k}$ falls off sufficiently fast for large $q^{2} / k^{2}$, and one may consider as an example $\sim \exp \left(-q^{2} / k^{2}\right)$. In lower dimensions it is also possible for some questions to use a masslike cutoff.

iv) It is not always possible to find a quadratic form $\Delta_{k} S$ (1.1) consistent with all symmetries of the theory. In our case this will be relevant for the Weylinvariance of the Liouville theory. The effective action $\Gamma_{k}$ will not exhibit all symmetries then. They are recovered only for $k \rightarrow 0$ where the "symmetry breaking term" $\Delta_{k} S$ vanishes. In consequence, the Ward identity for $\Gamma_{k}$ contains an additional $k$-dependent term which vanishes only for $k=0$. It is very important that the initial value $\Gamma_{\Lambda}$ is consistent with the anomalous Ward identity 5 - at least in a given approximation. Otherwise the trajectory in the space of actions described by the solution of the flow equation belongs to a different universality class within which the required symmetries are not preserved even for $k=0$.

Before embarking on the renormalization group equation and its solution, let us briefly recall some basic facts about Liouville theory which we shall need later on. We start from an arbitrary conformal field theory of central charge $c$ which is coupled to gravity and is governed by an action $S_{\text {matter }}\left[\Psi ; g_{\mu \nu}\right]$. (In bosonic string theory the matter fields $\Psi\left(x^{\mu}\right)$ correspond to the string positions $X^{m}\left(x^{\mu}\right), m=1, \ldots, c, \mu=1,2$.) Integrating out the matter field fluctuations,

$$
e^{-\Gamma_{\text {ind }}[g]}=\int \mathcal{D} \Psi e^{-S_{\text {matter }}[\Psi ; g]}
$$

leads to the induced gravity action 11]

$$
\Gamma_{\text {ind }}[g]=\frac{c}{96 \pi} I[g]+\lambda \int d^{2} x \sqrt{g}
$$

with

$$
I[g]=\int d^{2} x \sqrt{g} R \Delta_{g}^{-1} R
$$

where $\Delta_{g}$ is the Laplace-Beltrami operator of the metric $g_{\mu \nu}$ and $R$ is the curvature scalar. In a second step one has to integrate over the metric $g_{\mu \nu}$. We shall do this

\footnotetext{
${ }^{3}$ In a background field formalism the Ward identity may re replaced by an exact identity for the background dependence of $\Gamma_{k}[21]$.
} 
in the conformal gauge by picking a reference metric $\hat{g}_{\mu \nu}$ and writing

$$
g_{\mu \nu}(x)=e^{2 \phi(x)} \hat{g}_{\mu \nu}(x)
$$

We insert (1.6) into (1.4) and employ the identity

$$
I\left[e^{2 \sigma} \hat{g}_{\mu \nu}\right]=I\left[\hat{g}_{\mu \nu}\right]-4 \int d^{2} x \sqrt{\hat{g}}\left\{\hat{D}_{\mu} \sigma \hat{D}^{\mu} \sigma+\hat{R} \sigma\right\}
$$

After performing the integration over the ghost fields one is led to

$$
S[\phi ; \hat{g}]=-\frac{\kappa^{2}}{32 \pi} I[\hat{g}]+S_{L}[\phi ; \hat{g}]
$$

with the Liouville action

$$
S_{L}[\phi ; \hat{g}]=\frac{\kappa^{2}}{8 \pi} \int d^{2} x \sqrt{\hat{g}}\left\{\hat{D}_{\mu} \phi \hat{D}^{\mu} \phi+\hat{R} \phi+\frac{m^{2}}{2} e^{2 \phi}\right\}
$$

and

$$
3 \kappa^{2} \equiv 26-c
$$

Here $\hat{D}_{\mu}$ and $\hat{R}$ are constructed from $\hat{g}_{\mu \nu}$. The action $S[\phi ; \hat{g}]$ coincides with $\Gamma_{\text {ind }}\left[e^{2 \phi} \hat{g}\right]$ for $c$ replaced by $c-26$. This substitution takes care of the FaddeevPopov determinant related to the conformal gauge fixing. The parameter $m$ has dimension of mass, $m^{2} \equiv 16 \pi \lambda / \kappa^{2}$, and we assume $\kappa^{2}>0$ and $m^{2}>0$. What remains to be done is a functional integration over $\phi$.f

In this paper we consider (1.9) as the classical action of a field $\phi$ in a fixed background geometry $\hat{g}_{\mu \nu}$, and we try to quantize $\phi$ using the exact evolution equation. Because the decomposition (1.6) is invariant under the Weyl transformation

$$
\phi^{\prime}(x)=\phi(x)-\sigma(x), \quad \hat{g}_{\mu \nu}^{\prime}(x)=e^{2 \sigma(x)} \hat{g}_{\mu \nu}(x)
$$

the quantization should respect this "background split symmetry". This means that the functional integral over $\phi$ has to be performed with the Weyl invariant measure [30, [31], [32], [33], which derives from the following distance function in the space fields:

$$
d s_{\mathrm{Weyl}}^{2}=\int d^{2} x \sqrt{\hat{g}} e^{2 \phi(x)} \delta \phi(x)^{2}
$$

\footnotetext{
${ }^{4}$ The subsequent integration over the moduli implicit in $\hat{g}$ and the summation over the genus of the Riemann surface is outside the scope of this investigation.
} 
This measure is different from the translation-invariant measure which is used for usual scalar field theories. The latter is based upon the $\phi$-independent line element

$$
d s_{\text {trans }}^{2}=\int d^{2} x \sqrt{\hat{g}} \delta \phi(x)^{2}
$$

As discussed above, this means that the initial value $\Gamma_{\Lambda}$ should obey the modified Ward identity related to Weyl invariance.

Our paper is organized as follows. In section 2 we show in detail how the difference between the Weyl-invariant and the translation-invariant quantization manifests itself at the level of the exact renormalization group equations. We derive the Ward identities resulting from Weyl invariance and we use them to show that for $k \rightarrow 0$ the renormalization group flow converges towards a conformal field theory of central charge $26-c$. In section 3 we truncate the space of actions and use the Ward identities in order to determine the initial point of the evolution, $\Gamma_{\Lambda}$. It is one of the typical features of Liouville theory that in order to end up in the right universality class, $\Gamma_{\Lambda}$ cannot be chosen equal to the classical action $S_{L}$ (1.9). In section 4 we derive the truncated evolution equations. Their solution for $c \leq 1$ is discussed in section 5. In order to estimate the degree of precision which can be achieved with the truncation used, we compare the solution of the evolution equation to the constraints imposed by the Ward identities. Section 6 contains a brief comparison between Liouville theory and a free Feigin-Fuks theory. Section 7 is devoted to a general study of renormalization group fixed points. We show that the Liouville potential is singled out by the property that it is an eigenvector of the renormalization group flow linearized about the Gaussian fixed point. Our conclusions are presented in section 8. The overall picture emerging from this discussion is the conjecture that for low momenta the effective action $\Gamma_{k \rightarrow 0}$ of quantum Liouville theory has the same form as the classical action or at least very closely resembles the latter. Within certain approximations we find that $\Gamma$ equals $S_{L}$ of (1.9) with a renormalized parameter $m^{2}$. If exact, this would imply important "non-renormalization theorems", which go beyond the well-known statement [6] that the effective potential is essentially the same as the classical one. 


\section{Weyl Invariant Measure and Ward Identities}

Let $\chi\left(x^{\mu}\right)$ be an arbitrary scalar field in a background metric $g_{\mu \nu}$. 9 The derivation of the exact evolution equation [20] starts from the scale-dependent generating functional

$$
\begin{aligned}
e^{W_{k}[J ; g]}= & \int \mathcal{D}_{g} \chi \exp \left[-S[\chi ; g]+\int d^{2} x \sqrt{g} J(x) \chi(x)\right. \\
& \left.-\frac{1}{2} \int d^{2} x \sqrt{g} \chi(x) R_{k}\left(-D^{2}\right) \chi(x)\right]
\end{aligned}
$$

Here $\mathcal{D}_{g} \chi$ stands for either the Weyl or the translation invariant measure. The last term in the square bracket of (2.1) is a diffeomorphism-invariant infrared cutoff. The function $R_{k}\left(p^{2}\right)$ vanishes if the eigenvalues $p^{2}$ of $-D^{2}=-D_{\mu} D^{\mu}$ are much larger than the cutoff $k$, and it becomes a constant proportional to $k^{2}$ for $p^{2} \ll k^{2}$. The precise shape of the function $R_{k}\left(p^{2}\right)$ is not important, except that it has to interpolate monotonically between $R_{k}(\infty)=0$ and $R_{k}(0)=Z_{k} k^{2}$. (The meaning of the constant $Z_{k}$ will be explained later.) Often we shall write

$$
R_{k}\left(-D^{2}\right)=Z_{k} k^{2} C\left(-D^{2} / k^{2}\right)
$$

where $C$ is a dimensionless function with $C(0)=1$ and $C(\infty)=0$. (In some cases it is sufficient to use a constant mass like cutoff with $C \equiv 1$.) Expanding $\chi(x)$ in terms of eigenfunctions of $D^{2}$, we see that in $W_{k}$ (2.1) the high-frequency modes with covariant momenta $p^{2} \gg k^{2}$ are integrated out without any suppression whereas the low-frequency modes with $p^{2} \ll k^{2}$ are suppressed by a smooth, mass-type cutoff term $\sim k^{2} \chi^{2}$.

The effective average action $\Gamma_{k}[\phi ; g]$ is defined as the Legendre transform of $W_{k}[J ; g]$ with the infrared cutoff subtracted [20, [21]:

$$
\Gamma_{k}[\phi ; g]=\int d^{2} x \sqrt{g} \phi(x) J(x)-W_{k}[J ; g]-\frac{1}{2} \int d^{2} x \sqrt{g} \phi(x) R_{k}\left(-D^{2}\right) \phi(x)
$$

Here $J=J[\phi]$ has to be obtained by inverting the relation

$$
\phi(x) \equiv<\chi(x)>=[g(x)]^{-1 / 2} \frac{\delta W_{k}[J ; g]}{\delta J(x)}
$$

\footnotetext{
${ }^{5}$ From now on we write $g_{\mu \nu}$ for $\hat{g}_{\mu \nu}$ and omit the hats from $D_{\mu}$ and $R$.
} 
Our claim is that $\Gamma_{k}$ is a coarse-grained free energy or scale-dependent effective action which interpolates between the microscopic action $\Gamma_{\Lambda}$ and the usual effective action $\Gamma$ for $k=\Lambda$ and $k=0$, respectively. The equality $\Gamma_{k=0}=\Gamma$ is easy to establish: For $k=0$ the function $R_{k}\left(p^{2}\right)$ vanishes by construction for all $p^{2}$, and the cutoff term disappears from (2.1) and (2.3). On the other hand, the limit $\lim _{k \rightarrow \infty} \Gamma_{k}[\phi ; g]$ is more subtle. To investigate it, let us first note that $\Gamma_{k}$ obeys the following integral equation [29] which follows by inserting (2.3) into (2.1):

$$
\begin{aligned}
& e^{-\Gamma_{k}[\phi ; g]}=\int \mathcal{D}_{g} \chi \exp \left[-S[\chi ; g]+\int d^{2} x(\chi(x)-\phi(x)) \frac{\delta \Gamma_{k}[\phi ; g]}{\delta \phi(x)}\right] . \\
& \exp \left[-\frac{1}{2} \int d^{2} x \sqrt{g}(\chi(x)-\phi(x)) R_{k}\left(-D^{2}\right)(\chi(x)-\phi(x))\right]
\end{aligned}
$$

If $R_{k}$ is consistent with all symmetries, there is a heuristic argument that $\Gamma_{k \rightarrow \infty}$ equals the classical action: For $k \rightarrow \infty$ the function $R_{k}\left(p^{2}\right)$ approaches $Z_{k} k^{2}$ for any value of $p$. In this limit the second exponential in (2.5),

$$
\exp \left[-\frac{1}{2} Z_{k} k^{2} \int d^{2} x \sqrt{g}(\chi-\phi)^{2}\right]
$$

is proportional to a delta-functional $\delta(\chi-\phi)$. If the constant of proportionality is field-independent, we get indeed $\Gamma_{k \rightarrow \infty}=S$. Whether this is actually the case can be investigated by a saddle point approximation of the path-integral in (2.5). It becomes exact in the limit $k \rightarrow \infty$. One finds that $\Gamma_{k \rightarrow \infty}$ and $S$ differ by a fluctuation determinant in the heavy mass limit:

$$
\Gamma_{k \rightarrow \infty}=S+\frac{1}{2} \lim _{k \rightarrow \infty} \ln \operatorname{det}\left[S^{(2)}+Z_{k} k^{2}\right]
$$

$\left(S^{(2)}\right.$ denotes the Hessian of $S$.) In this limit, the fluctuation determinant gives rise to at most a couple of relevant and marginal operators such as $\phi \partial^{2} \phi, \phi^{2}$ and $\phi^{4}$ in $d=4$, say. These are precisely the terms which, in a renormalizable theory, one would have included in $S$ anyhow. Thus we can say that $\Gamma_{k \rightarrow \infty}=S$ holds true modulo a change of the bare parameters contained in $S$. For finite $\Lambda$ typical corrections beyond a shift in the bare parameters are suppressed by inverse powers of $\Lambda$. If this is the case one can ignore the difference between $\Gamma_{\Lambda}$ and $S$ for most practical purposes.

In Liouville theory the situation turns out to be more involved. In order to arrive at a quantum theory which is in the right universality class, namely 
that of conformal field theories with central charge $26-c$, some parameters in $\Gamma_{k \rightarrow \infty}$ have to be fine-tuned to a particular value. The situation is similar to the quantization of gauge theories. In order to obtain massless gauge fields for $k \rightarrow 0$, their mass at the UV cutoff must assume a precisely defined value. In the case of a Weyl invariant measure this will force us to use a $\Gamma_{\Lambda}$ which differs from $S$ in a well-defined way.

It is important to realize that in the renormalization group framework the starting point of the evolution, $\Gamma_{\Lambda}$, is the only place where the difference between the Weyl and the translation invariant measure enters. The evolution equation as such is the same in both cases. It is derived by taking a $k$-derivative of eq. (2.1) and reexpressing the result in terms of $\Gamma_{k}(2.3)$. Without making any assumption about $\mathcal{D}_{g} \chi$ one finds

$$
\frac{\partial}{\partial t} \Gamma_{k}[\phi ; g]=\frac{1}{2} \operatorname{Tr}\left[\left(\Gamma_{k}^{(2)}[\phi ; g]+R_{k}\left(-D^{2}\right)\right)^{-1} \frac{\partial}{\partial t} R_{k}\left(-D^{2}\right)\right]
$$

Here $t \equiv \ln k$ is the renormalization group "time" and $\Gamma_{k}^{(2)}$ and $R_{k}$ are differential operators in the variable $x$, related to the Hessian

$$
\Gamma_{k}^{(2)}(x, y)=[g(x) g(y)]^{-1 / 2} \frac{\delta^{2} \Gamma_{k}}{\delta \phi(x) \delta \phi(y)}
$$

by

$$
\Gamma_{k}^{(2)}(x, y)=\Gamma_{k}^{(2)} \delta(x-y) g(y)^{-1 / 2}
$$

As mentioned in the introduction, the quantization program consists of the following two steps: (i) Find the initial value $\Gamma_{\Lambda}$ for large $\Lambda$ for the classical theory under consideration. (ii) Solve the evolution equation (2.7) for this initial condition and let $k \rightarrow 0$ in the solution $\Gamma_{k}$.

Our tool to determine $\Gamma_{\Lambda}$ are the Ward identities related to the Weyl transformation (1.11) which we are going to derive now. We use the Liouville action $S=S_{L}$ of (1.9) in (2.1), and we subject both sides of eq. (2.1) to the transformation

$$
J^{\prime}=e^{-2 \sigma} J, \quad g_{\mu \nu}^{\prime}=e^{+2 \sigma} g_{\mu \nu}
$$

The integration variable on the r.h.s. of (2.1) is denoted $\chi^{\prime}$, and together with 
(2.10) we transform it according to

$$
\chi^{\prime}=\chi-\sigma
$$

Under the combined transformation the response of the Liouville action is

$$
S_{L}\left[\chi^{\prime} ; g^{\prime}\right]=S_{L}[\chi ; g]-\frac{\kappa^{2}}{8 \pi} \int d^{2} x \sqrt{g}\left\{D_{\mu} \sigma D^{\mu} \sigma+R \sigma\right\}
$$

which follows from $(\sqrt{g} R)^{\prime}=\sqrt{g}\left(R-2 D^{2} \sigma\right)$ and similar identities. By its very definition the Weyl invariant measure is invariant under the simultaneous transformation of $\chi$ and $g_{\mu \nu}$, but the translation invariant measure changes by the well-known Jacobian [11], 33] expressing the conformal anomaly:

$$
\mathcal{D}_{g^{\prime}} \chi^{\prime}=\mathcal{D}_{g} \chi \exp \left[\frac{\tau}{24 \pi} \int d^{2} x \sqrt{g}\left\{D_{\mu} \sigma D^{\mu} \sigma+R \sigma\right\}\right]
$$

Here $\tau=0(\tau=1)$ for the Weyl (translation) invariant measure. Though we are mainly interested in the Weyl invariant quantization, we treat both cases in parallel. Thus we are led to

$$
\begin{aligned}
W_{k}\left[e^{-2 \sigma} J, e^{2 \sigma} g\right] & =\frac{3 \kappa^{2}+\tau}{24 \pi} \int d^{2} x \sqrt{g}\left\{D_{\mu} \sigma D^{\mu} \sigma+R \sigma\right\} \\
-\int d^{2} x \sqrt{g} J \sigma & +\ln \int \mathcal{D}_{g} \chi \exp \left\{-S_{L}[\chi ; g]+\int d^{2} x \sqrt{g} J \chi\right. \\
& \left.-\frac{1}{2} \int d^{2} x \sqrt{g} e^{2 \sigma}(\chi-\sigma) R_{k}\left(-D_{g^{\prime}}^{2}\right)(\chi-\sigma)\right\}
\end{aligned}
$$

Expanding to first order in $\sigma$ one arrives at

$$
\begin{gathered}
2 \frac{g_{\mu \nu}}{\sqrt{g}} \frac{\delta W_{k}[J ; g]}{\delta g_{\mu \nu}(y)}-2 \frac{J(y)}{\sqrt{g}} \frac{\delta W_{k}[J ; g]}{\delta J(y)}=\frac{3 \kappa^{2}+\tau}{24 \pi} R(y)-J(y) \\
+<R_{k}\left(-D^{2}\right) \chi(y)>-<\chi(y) R_{k}\left(-D^{2}\right) \chi(y)> \\
-\operatorname{Tr}[\hat{R}(y)<\chi \otimes \chi>]
\end{gathered}
$$

with the $y$-dependent operator (acting on $x$ )

$$
\hat{R}_{k}(y)=\frac{g_{\mu \nu}(y)}{\sqrt{g(y)}} \frac{\delta}{\delta g_{\mu \nu}(y)} R_{k}\left(-D^{2}\left[g_{\mu \nu}(x)\right]\right)
$$


Eq. (2.15) can be reformulated as a statement about $\Gamma_{k}$ by using its definition (2.3) and exploiting the familiar property of the Legendre transform that the connected 2-point function

$$
G_{k}(x, y)=<\chi(x) \chi(y)>-\phi(x) \phi(y)
$$

is the matrix inverse of $\left(\Gamma_{k}^{(2)}+R_{k}\right)(x, y)$. The result is the following Weyl-Ward identity for the effective average action:

$$
\begin{aligned}
& 2 \frac{g_{\mu \nu}}{\sqrt{g}} \frac{\delta \Gamma_{k}[\phi ; g]}{\delta g_{\mu \nu}(x)}-\frac{1}{\sqrt{g}} \frac{\delta \Gamma_{k}[\phi ; g]}{\delta \phi(x)}=-\frac{3 \kappa^{2}+\tau}{24 \pi} R(x) \\
& \quad+<x\left|R_{k}\left(\Gamma_{k}^{(2)}+R_{k}\right)^{-1}\right| x>+\operatorname{Tr}\left[\hat{R}_{k}(x)\left(\Gamma_{k}^{(2)}(x)+R_{k}\right)^{-1}\right]
\end{aligned}
$$

This is a formula for the trace of the energy momentum tensor derived from $\Gamma_{k}$. For fields satisfying the equation of motion $\delta \Gamma_{k} / \delta \phi=0$ it consists of an anomaly term proportional to the curvature scalar, plus two additional pieces which are due to the explicit symmetry breaking by the cutoff. The Ward identity is consistent with the evolution equation in the sense that if the solution $\Gamma_{k}$ of (2.7) satisfies the Ward identity for one value of $k$, it automatically does so also for any other value. More precisely, this is true for exact solutions of the evolution equations. Approximate solutions, obtained for instance by truncating the space of all action functionals, are not necessarily consistent with the Ward identity. In this case the Ward identity is a powerful check for the quality of the truncation: a necessary condition for an approximation to be reliable is that (2.18) holds at the desired level of accuracy. 9

When an arbitrary (classical or quantum) conformal field theory with action $S$ and central charge $c[S]$ is coupled to gravity the trace of its energy momentum tensor

$$
T^{\mu \nu}[S] \equiv \frac{2}{\sqrt{g}} \frac{\delta S}{\delta g_{\mu \nu}}
$$

is (at least on shell) given by

$$
T_{\mu}^{\mu}[S]=-\frac{c[S]}{24 \pi} R+\text { const }
$$

\footnotetext{
${ }^{6}$ In the same spirit the modified Slavnov-Taylor identities where used to control truncations in Yang-Mills theory 28.
} 
The classical Liouville action (1.9) satisfies this condition with the central charge

$$
c\left[S_{L}\right]=3 \kappa^{2}=26-c
$$

Furthermore, by construction $R_{k}$ vanishes for $k \rightarrow 0$. Therefore the explicit symmetry-breaking terms in the Ward identity are absent in this limit, and (2.18) tells us that the evolution ends at a theory which is conformally invariant and has central charge

$$
c\left[\Gamma_{k \rightarrow 0}\right]=26-c+\tau
$$

For the Weyl-invariant measure $(\tau=0)$ this is the correct value. In order to investigate the implications of the Ward identity for $\Gamma_{k \rightarrow \infty}$ we have to make an ansatz for $\Gamma_{k}$. We shall discuss this in detail in section 3 .

Before closing this section we mention a slight generalization of the Ward identity. Let us couple the Liouville field in a Weyl-invariant way to a set of external fields $\psi_{i}(x)$ which are not to be quantized:

$$
S_{\psi}\left[\chi ; g, \psi_{i}\right]=\sum_{i} \int d^{2} x \sqrt{g} \psi_{i}(x) e^{2\left(1-\Delta_{i}^{0}\right) \chi}
$$

The action $S_{\psi}$ is invariant under

$$
\psi_{i}^{\prime}=e^{-2 \Delta_{i}^{0} \sigma} \psi_{i}
$$

together with (2.10) and (2.11). The $\psi_{i}$ 's could be some spin-0 primary fields of the underlying conformal theory of matter and ghost fields. Their bare dimensions are $\left(\Delta_{i}^{0}, \Delta_{i}^{0}\right)$. We shall investigate how these dimensions change when the system is coupled to quantized gravity ("gravitational dressing"). Replacing $S \equiv S_{L}$ by $S_{L}+S_{\psi}$ in the functional integral (2.1), the r.h.s. of the Ward identity remains unchanged, but on the l.h.s. we get an additional piece:

$$
\mathcal{L} \Gamma_{k}\left[\phi ; g, \psi_{i}\right]=-\frac{26-c+\tau}{24 \pi} R(x)+\mathcal{A}_{k}(x)
$$

In (2.25) we defined

$$
\mathcal{L} \equiv 2 \frac{g_{\mu \nu}(x)}{\sqrt{g}} \frac{\delta}{\delta g_{\mu \nu}(x)}-\frac{1}{\sqrt{g}} \frac{\delta}{\delta \phi(x)}-2 \sum_{i} \Delta_{i}^{0} \frac{\psi_{i}(x)}{\sqrt{g}} \frac{\delta}{\delta \psi_{i}(x)}
$$


and

$$
\mathcal{A}_{k}(x) \equiv<x\left|R_{k}\left(\Gamma_{k}^{(2)}+R_{k}\right)^{-1}\right| x>+\operatorname{Tr}\left[\hat{R}_{k}(x)\left(\Gamma_{k}^{(2)}+R_{k}\right)^{-1}\right]
$$

If $\mathcal{A}_{k}(x)$ contains $\phi$-dependent terms, the equation (2.25), even with $\phi$ and $\psi_{i}$ on shell, is not of the form (2.20) and $\Gamma_{k}$ is not a conformal theory, of course. We shall see that even for $k \rightarrow \infty$ such terms are actually present and prevent us from identifying $\Gamma_{k \rightarrow \infty}$ with the classical action.

The spacetime-integrated version of the Ward identity can be cast in a very suggestive form. It is not difficult to show that

$$
2 R_{k}+2 \int d^{2} y \sqrt{g(y)} \hat{R}_{k}(y)=\partial_{t} R_{k}+\eta_{k} R_{k}
$$

which expresses the fact that a Weyl transformation of $R_{k}$ with a constant $\sigma$ can be partially compensated for by a corresponding change of $k$. In (2.28) we introduced the anomalous dimension

$$
\eta_{k} \equiv-\partial_{t} \ln Z_{k}
$$

By using (2.28) in the integrated Ward identity and by eliminating the $\partial_{t} R_{k}$ term via the evolution equation (2.7) one arrives at

$$
\begin{aligned}
\partial_{t} \Gamma_{k}= & \int d^{2} x \sqrt{g} \mathcal{L} \Gamma_{k}+\frac{26-c+\tau}{24 \pi} \int d^{2} x \sqrt{g} R \\
& -\frac{1}{2} \eta_{k} \operatorname{Tr}\left[R_{k}\left(\Gamma_{k}^{(2)}+R_{k}\right)^{-1}\right]
\end{aligned}
$$

We shall see later on that the $\eta_{k}$ term is negligible actually. Thus (2.30) shows that (for $\phi$ on shell, $\delta \Gamma_{k} / \delta \phi=0$ ) the driving force for the evolution is the difference between $T_{\mu}^{\mu}\left[\Gamma_{k}\right]$ and the anomaly term for the central charge $26-c$ (for $\tau=0$ ). This makes it explicit that the evolution of $\Gamma_{k}$ will stop at a conformal theory with the right central charge.

\section{Truncation and Initial Value}

As it seems impossible to find exact solutions of the renormalization group equation, we try to construct an approximate solution by truncating the space of 
actions. The idea is to project the renormalization group flow on a subspace of the space of all action functionals. In our case this subspace has finite dimension and is coordinatized by a finite number of generalized couplings. Therefore the approximated trajectory $\left\{\Gamma_{k}, k \in[0, \Lambda]\right\}$ is described by several functions of $k$ which are the solution of a coupled system of ordinary differential equations. The difficult task is to guess (and to justify, in a second step) a truncation which leads to an approximate trajectory $\Gamma_{k}$ as close as possible to the true one. For scalar theories in $d>2$ dimensions, usually dimensional arguments can be used as a guide line. As long as anomalous dimensions are small, the importance of field monomials $\phi^{2}, \phi^{4}, \phi^{6}, \ldots$, say, may be judged on the basis of their canonical dimensions. In $d=2$ the situation is more involved since $\phi$ is dimensionless and at first sight all functions of $\phi$ seem equally important. (Canonically they are all marginal.) In the case at hand we shall make an ansatz for the truncated $\Gamma_{k}$ which is inspired by the form of the classical Liouville action. We solve the evolution equation using the truncation on the r.h.s.. We investigate explicitly if the general form of our ansatz remains approximately conserved by the flow. For this purpose we consider on the l.h.s. an extended class of actions with free functions instead of only a finite number of couplings. As a consistency check, we show that the resulting trajectory satisfies the Weyl-Ward identities to a very good approximation. More importantly, the Ward identity determines the correct initial value $\Gamma_{\Lambda}$ at the UV cutoff $\Lambda$.

We work within a general space of actions consisting of three pieces,

$$
\Gamma_{k}[\phi ; g, \psi]=\Gamma_{k}^{L}[\phi ; g]+\Gamma_{k}^{\psi}[\phi ; g, \psi]+\Gamma_{k}^{\text {grav }}[g]
$$

the most important one being

$$
\Gamma_{k}^{\mathrm{L}}[\phi ; g]=\frac{\kappa_{k}^{2}}{8 \pi} \int d^{2} x \sqrt{g}\left\{\zeta_{k}(\phi) D_{\mu} \phi D^{\mu} \phi+\omega_{k}(\phi) R+v_{k}(\phi)\right\}
$$

Here the running parameter $\kappa_{k}$ is defined as the coefficient of the $\phi R$-term by the convention

$$
\frac{\partial \omega_{k}}{\partial \phi}\left(\phi=\phi_{0}\right)=1
$$

where the reference point $\phi_{0}$ may be chosen conveniently, e.g. $\phi_{0}=0$. The truncation which we shall use on the r.h.s. of the flow equations and in the Ward 
identities assumes an effective potential of the form

$$
v_{k}(\phi)=\frac{m_{k}^{2}}{2 \alpha_{k}^{2} \kappa_{k}^{2}} e^{2 \alpha_{k} \kappa_{k} \phi}
$$

and

$$
\omega_{k}(\phi)=\phi, \quad \zeta_{k}(\phi)=\zeta_{k}
$$

In this case $\Gamma_{k}^{L}$ is parametrized by four functions of $k$, namely $\kappa_{k}, \zeta_{k}, m_{k}$ and $\alpha_{k}$, which have to be determined from the evolution equation. The second piece,

$$
\Gamma_{k}^{\psi}[\phi ; g, \psi]=\frac{1}{16 \pi} \sum_{i}\left(\frac{m_{i k}}{\alpha_{i k}}\right)^{2} \int d^{2} x \sqrt{g} \psi_{i} \exp \left(2 \alpha_{i k} \kappa_{k} \phi\right)
$$

is included in order to study the "gravitational dressing" of matter field operators $\psi_{i}(x)$. It is a scale-dependent analogue of $S_{\psi}$ in (2.23). We shall determine the functions $m_{i k}$ and $\alpha_{i k}$ from the evolution equation. This will enable us to compute the "dressed" scaling dimensions in presence of gravity [13], [34], [35]. The $\psi_{i}{ }^{\text {'s }}$ are treated in the external field approximation here. The third term in (3.1) is the $\phi$-independent pure gravity action for which we concentrate on the piece

$$
\Gamma_{k}^{\text {grav }}[g]=-\frac{\tilde{\kappa}_{k}^{2}}{32 \pi} I[g]
$$

This is the $k$-dependent counterpart of the term $-\left(\kappa^{2} / 32 \pi\right) I[\hat{g}]$ in the classical decomposition (1.8). Note that at the quantum level the functions $\kappa_{k}$ and $\tilde{\kappa}_{k}$ are different in general.

For the evolution equation and the Ward identity we need $\Gamma_{k}^{(2)}$, eq. (2.8), which is essentially the matrix of second functional derivatives with respect to $\phi$. From (3.2) with a $\phi$-independent coefficient $\zeta_{k}$ we see that an eigenmode of $-D^{2}$ with eigenvalue $p^{2}$ has the kinetic energy $\left(\zeta_{k} \kappa_{k}^{2} / 4 \pi\right) p^{2}$. According to the general rule [20], [21] we identify the constant $Z_{k}$ in the cutoff (2.2) with $\zeta_{k} \kappa_{k}^{2} / 4 \pi$, i.e.

$$
\begin{aligned}
R_{k}\left(-D^{2}\right) & =Z_{k} k^{2} C\left(-D^{2} / k^{2}\right) \\
Z_{k} & =\frac{1}{4 \pi} \zeta_{k} \kappa_{k}^{2}
\end{aligned}
$$

This makes sure that for the low momentum modes $(C \approx 1)$ the kinetic term and the cutoff combine to $Z_{k}\left(k^{2}+p^{2}\right)$, as it should be. In terms of $Z_{k}$ the second functional derivative reads

$$
\Gamma_{k}^{(2)}=Z_{k}\left(-D^{2}+E_{k}\right)
$$


with

$$
E_{k}=\tilde{m}_{k}^{2} \exp \left(2 \alpha_{k} \kappa_{k} \phi\right)+\sum_{i} \tilde{m}_{i k}^{2} \psi_{i} \exp \left(2 \alpha_{i k} \kappa_{k} \phi\right)
$$

where

$$
\tilde{m}_{k}^{2}=\frac{m_{k}^{2} \kappa_{k}^{2}}{4 \pi Z_{k}}=\frac{m_{k}^{2}}{\zeta_{k}} \quad, \quad \tilde{m}_{i k}^{2}=\frac{m_{i k}^{2} \kappa_{k}^{2}}{4 \pi Z_{k}}=\frac{m_{i k}^{2}}{\zeta_{k}} .
$$

Next we derive the constraints which the Ward identities impose on the functions $\kappa_{k}, \alpha_{k}, \ldots$ This information is needed to determine the correct initial value $\Gamma_{\Lambda}$. In section 2 the pure gravity term was not included. Hence $\Gamma_{k}$ of section 2 corresponds to $\Gamma_{k}^{L}+\Gamma_{k}^{\psi}$. Applying the differential operator (2.26) to the ansatz (3.2) with (3.4) and (3.5) yields

$$
\begin{aligned}
\mathcal{L}\left(\Gamma_{k}^{L}+\Gamma_{k}^{\psi}\right)= & \frac{\kappa_{k}^{2}}{4 \pi}\left(\zeta_{k}-1\right) D^{2} \phi-\frac{\kappa_{k}^{2}}{8 \pi} R \\
& +\frac{1}{8 \pi}\left(\frac{m_{k}}{\alpha_{k}}\right)^{2}\left(1-\alpha_{k} \kappa_{k}\right) e^{2 \alpha_{k} \kappa_{k} \phi} \\
& +\frac{1}{8 \pi} \sum_{i}\left(\frac{m_{i k}}{\alpha_{i k}}\right)^{2}\left(1-\Delta_{i}^{0}-\alpha_{i k} \kappa_{k}\right) \psi_{i} e^{2 \alpha_{i k} \kappa_{k} \phi}
\end{aligned}
$$

Similarly $\mathcal{A}_{k}$ has to be projected on the subspace of actions selected by the truncation. We make an ansatz containing the same field monomials as (3.13) and ignore all other invariants:

$$
\begin{aligned}
\mathcal{A}_{k}= & -\frac{\kappa_{k}^{2}}{4 \pi} \mathcal{A}_{k}^{(1)} D^{2} \phi+\frac{1}{24 \pi} \mathcal{A}_{k}^{(2)} R \\
& +\frac{1}{8 \pi} m_{k}^{2} \mathcal{A}_{k}^{(3)} e^{2 \alpha_{k} \kappa_{k} \phi} \\
& +\frac{1}{8 \pi} \sum_{i} m_{i k}^{2} \mathcal{A}_{k}^{(4)} \psi_{i} e^{2 \alpha_{i k} \kappa_{k} \phi}+\ldots
\end{aligned}
$$

In this manner the Ward identities boil down to the following conditions which should hold for all values of $k$ :

$$
\begin{gathered}
\zeta_{k}=1-\mathcal{A}_{k}^{(1)} \\
3 \kappa_{k}^{2}=26-c+\tau-\mathcal{A}_{k}^{(2)} \\
\alpha_{k} \kappa_{k}+\alpha_{k}^{2} \mathcal{A}_{k}^{(3)}-1=0 \\
\alpha_{i k} \kappa_{k}+\alpha_{i k}^{2} \mathcal{A}_{k}^{(4)}+\Delta_{i}^{0}-1=0
\end{gathered}
$$


In general the coefficients $\mathcal{A}_{k}^{(n)}$ depend on the choice for the cutoff function $C$. In order to determine the initial conditions for large $\Lambda$ we need their values only for $k \rightarrow \infty$. In this limit all cutoffs $C\left(-D^{2} / k^{2}\right)$ become equivalent to the mass-like cutoff $C \equiv 1$. For later use we compute the $\mathcal{A}_{k}^{(n)}$ 's also for finite values of $k$, but for simplicity we restrict ourselves to $C \equiv 1$. Note that $\hat{R}_{k} \equiv 0$ in this case. The terms proportional to the curvature scalar and to $D^{2} \phi$ are most easily extracted from $\mathcal{A}_{k}(x)$ by writing

$$
\mathcal{A}_{k}(x)=k^{2} \int_{0}^{\infty} d s e^{-s k^{2}}<x\left|e^{s\left(D^{2}-E_{k}\right)}\right| x>
$$

with $E_{k}(x)=\tilde{m}_{k}^{2}\left(1-2 \alpha_{k} \kappa_{k} \phi+\ldots\right)$ and by using the standard heat kernel expansion

$$
<x\left|e^{s\left(D^{2}+P(x)\right)}\right| x>=\frac{1}{4 \pi s}\left[1+\frac{1}{6} R s+\frac{1}{6} D^{2} P s^{2}+\ldots\right]
$$

Thus one finds

$$
\begin{gathered}
\mathcal{A}_{k}^{(1)}=\frac{\alpha_{k}}{3 \kappa_{k}} \frac{k^{2} \tilde{m}_{k}^{2}}{\left(\tilde{m}_{k}^{2}+k^{2}\right)^{2}} \\
\mathcal{A}_{k}^{(2)}=\frac{k^{2}}{\tilde{m}_{k}^{2}+k^{2}}
\end{gathered}
$$

In order to determine $\mathcal{A}_{k}^{(3)}$ and $\mathcal{A}_{k}^{(4)}$ we may set $\phi, \psi_{i}=$ const, $g_{\mu \nu}=\delta_{\mu \nu}$ such that

$$
\mathcal{A}_{k}(x)=\int \frac{d^{2} p}{(2 \pi)^{2}} \frac{k^{2}}{p^{2}+k^{2}+E_{k}}
$$

The result depends on the relative size of $k^{2}$ and $E_{k}$. For $k^{2} \gg E_{k}$ we can expand in $E_{k}$ and find up to a constant

$$
\mathcal{A}_{k}=-E_{k} \int \frac{d^{2} p}{(2 \pi)^{2}} \frac{k^{2}}{\left(p^{2}+k^{2}\right)^{2}}=-\frac{m_{k}^{2}}{4 \pi \zeta_{k}} \exp \left(2 \alpha_{k} \kappa_{k} \phi\right)-\sum_{i} \frac{m_{i k}^{2}}{4 \pi \zeta_{k}} \psi_{i} \exp \left(2 \alpha_{i k} \kappa_{k} \phi\right)
$$

For this region, which is relevant for $k=\Lambda$, one can extract

$$
\mathcal{A}_{k}^{(3)}=A_{k}^{(4)}=-2 \zeta_{k}^{-1}
$$

On the other hand, for small $k$ we have to use the general answer

$$
\mathcal{A}_{k}(\phi)-\mathcal{A}_{k}(\phi \rightarrow-\infty)=-\frac{k^{2}}{4 \pi} \ln \frac{k^{2}+E_{k}}{k^{2}}
$$

which vanishes for $k \rightarrow 0$ as it should be. We infer $\mathcal{A}_{k \rightarrow 0}^{(3)}=\mathcal{A}_{k \rightarrow 0}^{(4)}=0$. 
Let us now discuss what this implies for the initial point of the evolution $\Gamma_{\Lambda}$. Letting $k \rightarrow \infty$ we obtain at the UV cutoff $\Lambda$

$$
\mathcal{A}_{\Lambda}^{(1)}=0, \quad \mathcal{A}_{\Lambda}^{(2)}=1, \quad \mathcal{A}_{\Lambda}^{(3)}=\mathcal{A}_{\Lambda}^{(4)}=-2 \zeta_{\Lambda}^{-1}
$$

By (3.15) with (3.27) the initial wave function normalization constant is fixed to be unity,

$$
\zeta_{\Lambda}=1
$$

such that $\mathcal{A}_{\Lambda}^{(3)}=\mathcal{A}_{\Lambda}^{(4)}=-2$. By $(\underline{3.16})$ the initial value for the coefficient of the $R \phi$-term in the action is

$$
3 \kappa_{\Lambda}^{2}=25-c+\tau
$$

In the classical action $S_{L}$ the corresponding coefficient of $R \phi / 24 \pi$ is $c\left[S_{L}\right]=3 \kappa^{2}=$ $26-c$. For the translation invariant quantization $(\tau=1)$ these values coincide, but for the Weyl invariant case $(\tau=0)$ we have to start from a different value, $3 \kappa_{\Lambda}^{2}=25-c$, and $\Gamma_{\Lambda}^{L}$ cannot coincide with $S_{L}$. We emphasize that $3 \kappa_{\Lambda}^{2}$ does not have the interpretation of a central charge if $\Gamma_{\Lambda}$ does not describe a conformal field theory. As we remarked at the end of section 2 , any $\phi$-dependence of $\mathcal{A}_{\Lambda}$ means that the trace of the energy momentum tensor of $\Gamma_{\Lambda}$ does not have the form (2.20). The nonzero values of $\mathcal{A}_{\Lambda}^{(3)}$ and $\mathcal{A}_{\Lambda}^{(4)}$ imply that for $m_{\Lambda}, m_{i \Lambda} \neq 0$ such terms are indeed present in $T_{\mu}^{\mu}\left[\Gamma_{\Lambda}\right]$. In the exceptional case $m_{\Lambda}, m_{i \Lambda}=0, \Gamma_{\Lambda}$ is a conformal free field theory of the Feigin-Fuks type [36 with central charge $25-c+\tau$.

From (3.17) we also obtain a relation which determines the initial value $\alpha_{\Lambda}$ in terms of $\kappa_{\Lambda}$,

$$
\alpha_{\Lambda} \kappa_{\Lambda}=1+2 \alpha_{\Lambda}^{2}
$$

With $\tau=0$ for the Weyl-invariant measure we have the two solutions

$$
\alpha_{\Lambda}=\frac{1}{4 \sqrt{3}}[\sqrt{25-c} \pm \sqrt{1-c}]
$$

with the perturbative branch $\left(\alpha_{\Lambda} \kappa_{\Lambda}=1+0(1 / c)\right.$ for $\left.c \rightarrow-\infty\right)$, corresponding to the minus sign. The starting value of the coefficient in the Liouville exponential is fixed to be

$$
\alpha_{\Lambda} \kappa_{\Lambda}=\frac{1}{12}[25-c \pm \sqrt{(c-25)(c-1)}]
$$


It is real only for $c \leq 1$ and $c \geq 25$. In the following we restrict ourselves to $c \leq 1$. Eq. (3.32) is familiar from other approaches to Liouville theory [33]. In the present context its interpretation is different, however. Likewise eq. (3.18) yields

$$
2 \alpha_{i \Lambda}^{2}-\alpha_{i \Lambda} \kappa_{\Lambda}-\Delta_{i}^{0}+1=0
$$

which fixes $\alpha_{i \Lambda}$ in terms of $\Delta_{i}^{0}$ and $\kappa_{\Lambda}$. Knowing $\alpha_{i k}$ and $\alpha_{k}$ at a certain scale $k$ is equivalent to knowing the gravitationally dressed scaling dimension $\Delta_{i}$ of $\psi_{i}$. By definition this dimension describes the scaling of $\psi_{i}$ relative to the one of the area operator which is governed by $\alpha_{k}$. One defines [30], [34] $\Delta_{i}(k) \equiv 1-\alpha_{i k} / \alpha_{k}$, and obtains from (3.33) for $k=\Lambda$ and the sign corresponding to the perturbative branch

$$
\Delta_{i}(\Lambda)=\frac{\sqrt{\kappa_{\Lambda}^{2}-8+8 \Delta_{i}^{0}}-\sqrt{\kappa_{\Lambda}^{2}-8}}{\kappa_{\Lambda}-\sqrt{\kappa_{\Lambda}^{2}-8}}
$$

Or, with $\tau=0$,

$$
\Delta_{i}(\Lambda)=\frac{\sqrt{1-c+24 \Delta_{i}^{0}}-\sqrt{1-c}}{\sqrt{25-c}-\sqrt{1-c}}
$$

The r.h.s. of this expression is precisely what appears in the famous KPZ-formula 113 for the scaling dimension of $\psi_{i}$ in presence of quantized gravity. However, in the renormalization group framework the properties of the quantum theory are obtained for $k \rightarrow 0$ rather than at $k=\Lambda$. Therefore (3.35) is not the KPZ-formula yet. It obtains only provided we can show that $\Delta_{i}(0)=\Delta_{i}(\Lambda)$.

Finally we have to fix the initial value $\tilde{\kappa}_{\Lambda}$ in the pure gravity piece (3.7). This term was not included when we derived the Ward identity in section 2 because it can be pulled in front of the path integral (2.1). $\mathcal{A}_{k \rightarrow \infty}$ produces no term proportional to $I[g]$, so we may read off $\tilde{\kappa}_{\Lambda}$ from the classical action (1.8) with (1.10):

$$
3 \tilde{\kappa}_{\Lambda}^{2}=26-c
$$

As $\kappa_{\Lambda} \neq \tilde{\kappa}_{\Lambda}$ for $\tau=0, \Gamma_{\Lambda}^{L}$ and $\Gamma_{\Lambda}^{\text {grav }}$ cannot be combined into a functional of $g_{\mu \nu} e^{2 \phi}$, and $\Gamma_{\Lambda}$ is clearly not Weyl-invariant. This lack of Weyl invariance compensates for the one arising from the infrared cutoff term $\Delta_{\Lambda} S$. Weyl invariance is restored when all fluctuations are integrated out at $k=0$. 
Our method of fixing the initial condition for the Weyl-invariant quantization has a certain similarity with the approach of Distler and Kawai [30 where an ansatz is made for the Jacobian relating the Weyl- to the translation-invariant measure and where Weyl-Ward identities (different from ours) are used to fix the free parameters [32]. This similarity is accidental, however, because we happend to use a truncation for $\Gamma_{k}$ which is similar to the classical Liouville action. Our method works for more general truncations in the same way. The Ward identity picks the "best" initial point from the subspace of truncated actions. It is not necessary here to assume that the Jacobian has the classical Liouville form.

To summarize this section, the initial value $\Gamma_{\Lambda}$ is given by eq. (3.1), with $\zeta_{\Lambda}=1,3 \kappa_{\Lambda}^{2}=25-c, 3 \widetilde{\kappa}_{\Lambda}^{2}=26-c$ and $\alpha_{\Lambda}, \alpha_{i \Lambda}$ specified by (3.32) and (3.33). This holds for $\Lambda \rightarrow \infty$. Since $\Lambda$ has the dimension of a mass, the precise meaning of the last statement involves the ratio $\Lambda / m$. It will be specified in the next section.

\section{The Evolution Equations}

In this section we derive the coupled system of ordinary differential equations for the functions $\alpha_{k}, m_{k}, \ldots$ appearing in the truncation ansatz. In order to investigate the universality properties of the various $\beta$-functions, we employ an unspecified cutoff function $C$. Upon inserting the truncation (3.1), its Hessian (3.10) and the cutoff (3.8) into the renormalization group equation (2.7) we have to evaluate

$$
\partial_{t} \Gamma_{k}=B_{k}-\frac{1}{2} \eta_{k} B_{k}^{(\eta)}
$$

with (a prime denotes the derivative with respect to the argument):

$$
\begin{gathered}
B_{k} \equiv \operatorname{Tr}\left[\left(k^{2} C\left(-D^{2} / k^{2}\right)+D^{2} C^{\prime}\left(-D^{2} / k^{2}\right)\right)\left(-D^{2}+k^{2} C\left(-D^{2} / k^{2}\right)+E_{k}\right)^{-1}\right] \\
B_{k}^{(\eta)} \equiv \operatorname{Tr}\left[k^{2} C\left(-D^{2} / k^{2}\right)\left(-D^{2}+k^{2} C\left(-D^{2} / k^{2}\right)+E_{k}\right)^{-1}\right]
\end{gathered}
$$

Here

$$
\eta_{k} \equiv-\partial_{t} \ln Z_{k}=-\partial_{t} \ln \left(\kappa_{k}^{2} \zeta_{k}\right)
$$

is the anomalous dimension of the Liouville field. 
The projected evolution equation (4.1) is to be understood as follows. Its l.h.s. is given by the ansatz (3.1) with $\kappa_{k}^{2}$ replaced by $\partial_{t} \kappa_{k}^{2}$, etc.. When the r.h.s. is expanded in a basis of invariants constructed from $\phi, g_{\mu \nu}$ and $\psi_{i}$, only the invariants also present on the l.h.s. should be retained. The projection on the subspace means that we discard all other terms. The evolution equations for $\kappa_{k}^{2}, \ldots$ obtain by comparing the coefficients of the respective invariants on both sides of eq. (4.1). The coefficient of the $R \phi$-term determines the running of $\kappa_{k}^{2}$. Once it is known, the prefactor of $\left(D_{\mu} \phi\right)^{2}$ gives us the evolution of $\zeta_{k}$. This method is meaningful only if the invariants are linearly independent in an appropriate sense. This is certainly the case for the "kinetic" terms $\left(D_{\mu} \phi\right)^{2}$ and $R \phi$. They are the first terms in an expansion in the number of derivatives and powers of the curvature tensor, and they could be extended to a complete basis in the full space by adding all terms of the type $\left(D^{2} \phi\right)^{2}, R \phi^{2},\left(D^{2} \phi\right) R \phi, \cdots$. For the potential $v_{k}(\phi)$ the situation is slightly more complicated. In the spirit of a Laplace or a Fourier transform we use the exponentials $\exp (\gamma \phi)$ as basis vectors and consider them independent for different values of $\gamma$. In the truncation only a single basis vector is retained, the one with $\gamma=2 \alpha_{k} \kappa_{k}$. This basis vector is allowed to change with $k$. Finally we assume that the set $\left\{\psi_{i}\right\}$ is linearly independent in the above sense and does not contain the identity operator. Hence $\exp \left(2 \alpha_{k} \kappa_{k} \phi\right)$ and $\psi_{i} \exp \left(2 \alpha_{i k} \kappa_{k} \phi\right)$ are also independent.

For a more general investigation we will occasionally consider on the l.h.s. an enlarged ansatz with arbitrary functions $v_{k}(\phi), \zeta_{k}(\phi)$ and $\omega_{k}(\phi)$ in eq. (3.2). This allows us to study to what extent invariants beyond our truncation are generated by the evolution. It is in this generalized context that we will see that the exponential form of the potential does not change.

As we expect $\eta_{k}$ to be small, we ignore the term in (4.1) proportional to $\eta_{k}$ in a first approximation and solve only $\partial_{t} \Gamma_{k}=B_{k}$. This procedure will prove consistent, because for the resulting solution $\eta_{k}$ is indeed negligible. More precisely, we shall see that $Z_{k}$ varies between $k=\Lambda$ and $k=0$ only by a finite amount even in the limit when $\Lambda$ is sent to infinity. We will see below that this change occurs for $k^{2} \approx \tilde{m}^{2}$ which shows that $\eta_{k}$ vanishes both for $k^{2} \gg \tilde{m}^{2}$ and $k^{2} \ll \tilde{m}^{2}$. 
We start with the term $\sim \int d^{2} x \sqrt{g} R \omega_{k}(\phi)$ generated by $B_{k}$. Because it contains no $\psi_{i}$ and no derivatives of $\phi$, we may set $\psi_{i}(x) \equiv 0$ and $\phi(x)=\phi=$ const for its evaluation. Then $E_{k}(x)$, (3.11), becomes independent of $x$. If we take advantage of the heat-kernel expansion (3.20), we see that

$$
\operatorname{Tr}\left[f\left(-D^{2}\right)\right]=\frac{1}{24 \pi} \int d^{2} x \sqrt{g} R f(0)+\ldots
$$

for every function of the scalar Laplacian which can be written as a Laplace or Fourier transform: $f\left(-D^{2}\right)=\int_{0}^{\infty} d s \tilde{f}(s) \exp \left(s D^{2}\right)$. As a result,

$$
B_{k}=\frac{1}{24 \pi} \int d^{2} x \sqrt{g} R \frac{k^{2} C(0)}{k^{2} C(0)+E_{k}}+\text { other invariants }
$$

Recall that by definition $C(0)$ equals unity for any choice of the cutoff function. We see that the coefficient of the $\sqrt{g} R$ term, and hence the $\beta$-function for $\kappa_{k}^{2}$, is universal in the sense that it does not depend on the form of the cutoff. More explicitly, we find the flow equation (omitting occasionally the subscripts $k$ for the couplings)

$$
\partial_{t}\left(\kappa^{2} \omega(\phi)\right)=\frac{1}{3} \frac{k^{2}}{k^{2}+\tilde{m}^{2} \exp (2 \alpha \kappa \phi)}
$$

For example, an expansion around $\phi=0$ yields

$$
\partial_{t}\left(\kappa^{2} \omega(0)\right)=\frac{1}{3} \frac{k^{2}}{k^{2}+\tilde{m}^{2}}
$$

and, with the reference point $\phi_{0}=0$ in $(3.3)$,

$$
\partial_{t} \kappa^{2}=\partial_{t}\left(\kappa^{2} \omega^{\prime}(0)\right)=-\frac{2}{3} \alpha \kappa \frac{k^{2} \tilde{m}^{2}}{\left(k^{2}+\tilde{m}^{2}\right)^{2}}
$$

On the other hand, for $\phi$ much smaller than a critical value $\phi_{c}$

$$
\phi_{c}(k) \equiv \frac{1}{2 \alpha_{k} \kappa_{k}} \ln \frac{k^{2}}{\tilde{m}_{k}^{2}}
$$

one has

$$
\partial_{t}\left(\kappa^{2} \omega\left(\phi \ll \phi_{c}\right)\right)=\frac{1}{3}\left(1-\frac{\tilde{m}^{2}}{k^{2}} \exp (2 \alpha \kappa \phi)\right)
$$

Qualitatively, one finds that $\kappa^{2} \omega(\phi)$ changes at a constant rate $\partial_{t}\left(\kappa^{2} \omega\right)=\frac{1}{3}$ for $\phi \ll \phi_{c}$ whereas the evolution stops due to suppression factors for $\phi \gg \phi_{c}$ :

$$
\partial_{t}\left(\kappa^{2} \omega\left(\phi \gg \phi_{c}\right)\right)=\frac{1}{3} \exp \left(-2 \alpha \kappa\left(\phi-\phi_{c}\right)\right)
$$


Next we consider the evolution of $\zeta_{k}(\phi)$ and $v_{k}(\phi)$. These pieces can be extracted from the flow of $\Gamma_{k}$ for $g_{\mu \nu}=\delta_{\mu \nu}$. This part of the investigation is therefore completely parallel to a two-dimensional scalar theory in flat space [18], [23, 27, as given by

$$
\Gamma_{k}[\phi]=\int d^{2} x\left\{\frac{1}{2} Z_{k}(\phi) \partial_{\mu} \phi \partial^{\mu} \phi+U_{k}(\phi)\right\}
$$

with effective potential

$$
U_{k}(\phi) \equiv \frac{\kappa_{k}^{2}}{8 \pi} v_{k}(\phi)
$$

and "wave function renormalization"

$$
Z_{k}(\phi) \equiv \frac{\kappa_{k}^{2}}{4 \pi} \zeta_{k}(\phi)
$$

We start with the evolution of the effective potential $U_{k}$. In a first step we keep on the l.h.s. of the flow equation an arbitrary function of $\phi$ whereas on the r.h.s. the ansatz (3.4)

$$
U_{k}(\phi)=\frac{\tilde{m}_{k}^{2} Z_{k}}{\left(2 \alpha_{k} \kappa_{k}\right)^{2}} \exp \left(2 \alpha_{k} \kappa_{k} \phi\right)
$$

is inserted. This allows us to investigate if terms different from (4.16) are generated in the course of the evolution, i.e., if the effective potential changes away from its form of a simple exponential. For a computation of the flow $\partial_{t} U_{k}$ we set $\psi_{i}=0, \phi=$ const. Hence the trace over functions of $D^{2}=\partial^{2}$ becomes a simple momentum integral. The evolution of $U_{k}$ is therefore given by the standard flow equations for a two-dimensional scalar theory [18], [23], [27]. What is special, however, is our ansatz for the potential on the r.h.s. for which one finds

$$
\partial_{t} U(\phi)=\frac{k^{2}}{4 \pi} \int_{0}^{\infty} d y\left(C(y)-y C^{\prime}(y)\right)\left[y+C(y)+\frac{\tilde{m}^{2}}{k^{2}} \exp (2 \alpha \kappa \phi)\right]^{-1}
$$

The global behavior of $U$ can be understood by noting that $C(y)-y C^{\prime}(y)=$ $\frac{\partial}{\partial k^{2}}\left(k^{2} C\left(\frac{x}{k^{2}}\right)\right)$ is positive for all $y=x / k^{2}$. For a fixed value of $\phi$ therefore $U_{k}(\phi)$ always decreases as $k \rightarrow 0$. This decrease is faster for small $\phi$ than for large $\phi$ and the rate of decrease depends monotonically on $\phi$. We see that $U_{k}(\phi)$ remains monotonically increasing with $\phi$ in the whole range of $\phi$ and can therefore not develop a minimum for finite $\phi$ ! (The minimum remains at $\phi \rightarrow-\infty$ ). Actually, 
this remark generalizes within the truncation (3.2) for arbitrary monotonically increasing potentials with monotonically increasing second derivatives. Our finding that quantum Liouville theory does not have a ground state with finite constant $\phi$ seems therefore rather robust. The more quantitative behavior depends crucially on whether $\phi$ is smaller or larger than $\phi_{c}$ : For $\phi \gg \phi_{c}$ the running is exponentially suppressed

$$
\partial_{t} U\left(\phi \gg \phi_{c}\right)=\frac{k^{2}}{4 \pi} \exp \left[-2 \alpha \kappa\left(\phi-\phi_{c}\right)\right] \int_{0}^{\infty} d y\left(C(y)-y C^{\prime}(y)\right)
$$

In the opposite region $\phi \ll \phi_{c}$ we can expand in the mass-like term

$$
\partial_{t} U\left(\phi \ll \phi_{c}\right)=\frac{l_{0}}{4 \pi} k^{2}-\frac{l_{1}}{4 \pi} E_{k}=\frac{l_{0}}{4 \pi} k^{2}-\frac{\tilde{m}^{2}}{4 \pi} \exp (2 \alpha \kappa \phi)
$$

with

$$
l_{0} \equiv \int_{0}^{\infty} d y \frac{C(y)-y C^{\prime}(y)}{y+C(y)}
$$

It is remarkable that the momentum integral' $\left(y \equiv p^{2} / k^{2}\right)$

$$
l_{1} \equiv \int_{0}^{\infty} d y \frac{C(y)-y C^{\prime}(y)}{(y+C(y))^{2}}=1
$$

is again independent of the detailed form of $C(y)$. The integrand is a total derivative and it is sufficient to know that $C(0)=1$ in order to obtain the universal value $l_{1}=1$. In consequence, for $\phi \ll \phi_{c}$ we find that the evolution generates in $U_{k}$ only a contribution proportional to the coefficient multiplying $\exp (2 \alpha \kappa \phi)$ plus a constant term. Defining the constant

$$
\lambda_{L}=U(\phi \rightarrow-\infty)
$$

one easily finds the solution

$$
\lambda_{L}(k)=\lambda_{L}(\Lambda)-\frac{l_{0}}{2 \pi}\left(\Lambda^{2}-k^{2}\right)
$$

Subtracting $\lambda_{L}$ we see that for $\phi \ll \phi_{c}$ the exponential shape of $U$ does not change in the course of the evolution . This implies $\partial_{t}(\alpha \kappa)=0$, i.e., the product $\alpha_{k} \kappa_{k}$ is a renormalization group invariant:

$$
\alpha_{k} \kappa_{k}=\text { const }=\alpha_{\Lambda} \kappa_{\Lambda}
$$

\footnotetext{
${ }^{7}$ The integrals $l_{0}, l_{1}$ belong to the standard integrals $l_{n}^{d}$ for $d=2$ appearing in [18, 23.
} 
As a result, (4.19) boils down to an evolution equation for $\tilde{m}^{2}$

$$
\partial_{t} \tilde{m}^{2}=\left[\eta-\frac{(\alpha \kappa)^{2}}{\pi} \frac{1}{Z}\right] \tilde{m}^{2}=\left[\eta-\frac{4 \alpha^{2}}{\zeta}\right] \tilde{m}^{2}
$$

We recall, however, that (4.24) and (4.25) become exact only for $\phi \ll \phi_{c}$. For $\phi \gg \phi_{c}$ the running of $U_{k}$ stops according to eq. (4.18) and $\partial_{t}\left(Z \tilde{m}^{2}\right)$ tends therefore to zero if the parametrization (4.16) is used in this range of $\phi$. This can be seen explicitly by applying a derivative $\partial_{t}$ to the definition

$$
Z \tilde{m}^{2}=\frac{\kappa^{2}}{4 \pi} m^{2}=\frac{\partial^{2} U}{\partial \phi^{2}}(0)
$$

and by using (4.17):

$$
\begin{aligned}
\partial_{t}\left(Z \tilde{m}^{2}\right) & =-\frac{\alpha^{2} \kappa^{2}}{\pi} \tilde{m}^{2} \mathcal{J}\left(\frac{\tilde{m}^{2}}{k^{2}}\right) \\
\mathcal{J}\left(\frac{\tilde{m}^{2}}{k^{2}}\right) & \equiv \int_{0}^{\infty} d y\left(C-y C^{\prime}\right)\left(y+C-\frac{\tilde{m}^{2}}{k^{2}}\right)\left(y+C+\frac{\tilde{m}^{2}}{k^{2}}\right)^{-3}
\end{aligned}
$$

For the special case $C=1$ this yields

$$
\partial_{t}\left(Z \tilde{m}^{2}\right)=-\frac{\alpha^{2} \kappa^{2}}{\pi} \frac{k^{4} \tilde{m}^{2}}{\left(k^{2}+\tilde{m}^{2}\right)^{2}}
$$

If we treat the exponentials multiplied by $\psi_{i}$ in the same manner we obtain in the region $\phi \ll \phi_{c}$ relations similar to (4.24) and (4.25) with $\alpha_{k}$ and $m_{k}$ replaced by $\alpha_{i k}$ and $m_{i k}$, respectively. This means that the gravitationally modified scaling dimension of $\psi_{i}$ does not get renormalized in this region

$$
\Delta_{i}(k)=1-\frac{\alpha_{i k}}{\alpha_{k}}=1-\frac{\alpha_{i \Lambda}}{\alpha_{\Lambda}}=\Delta_{i}(\Lambda)
$$

Here the initial value $\Delta_{i}(\Lambda)$ is related to the classical dimension $\Delta_{i}^{0}$ by eq. (3.35), a consequence of the Ward identity. Again, for $\phi \gg \phi_{c}$ the running stops effectively.

In order to determine the flow of $Z_{k}$, we have to extract the $\left(\partial_{\mu} \phi\right)^{2}$ piece from $B_{k}$, evaluated for $g_{\mu \nu}=\delta_{\mu \nu}, \psi_{i}=0$. Using standard derivative expansion techniques, this can be done in complete analogy to ref. [18], 23] and one obtains $\left(x \equiv p^{2}\right)$

$$
\partial_{t} Z(\phi)=\frac{1}{8 \pi}\left(\frac{\partial^{3} U}{\partial \phi^{3}}\right)^{2} \int_{0}^{\infty} d x x \tilde{\partial}_{t}\left\{\left(\frac{\partial P}{\partial x}\right)^{2}\left(P+\frac{\partial^{2} U}{\partial \phi^{2}}\right)^{-4}\right\}
$$

\footnotetext{
${ }^{8}$ The r.h.s. of eq. 4.30 corresponds to $-\tilde{\eta} Z_{k}$ in [18, 23].
} 
where

$$
P(x) \equiv Z_{k} x+R_{k}(x)=Z_{k}\left[x+k^{2} C\left(\frac{x}{k^{2}}\right)\right]
$$

and $\tilde{\partial}_{t}$ is a logarithmic $k$-derivative acting only on $R_{k}$, i.e., $\tilde{\partial}_{t}=\partial_{t} R_{k}\left(\partial / \partial R_{k}\right)$. For $\phi \ll \phi_{c}$ the running of $Z(\phi)$ is suppressed by the exponential factor $\left(\partial^{3} U / \partial \phi^{3}\right)=$ $2 \alpha \kappa \tilde{m}^{2} Z \exp (2 \alpha \kappa \phi)$ according to

$$
\partial_{t} Z\left(\phi \ll \phi_{c}\right)=-\frac{m_{4}}{\pi} \alpha^{2} \kappa^{2} \frac{\tilde{m}^{4}}{k^{4}} \exp (4 \alpha \kappa \phi)
$$

where the constant $m_{4}$ is given by

$$
m_{4}=\int_{0}^{\infty} d y y^{-2} \frac{\partial}{\partial y}\left\{\left(1+C^{\prime}(y)\right)^{2} y^{4}(y+C(y))^{-4}\right\}
$$

On the other hand, the running is also suppressed for $\phi \gg \phi_{c}$ due to the large mass term $\partial^{2} U / \partial \phi^{2}=\tilde{m}^{2} Z \exp (2 \alpha \kappa \phi)$,

$$
\partial_{t} Z\left(\phi \gg \phi_{c}\right)=-\frac{2 \alpha^{2} \kappa^{2}}{\pi} \frac{k^{4}}{\tilde{m}^{4}} \exp (-4 \alpha \kappa \phi) \int_{0}^{\infty} d y y^{2}\left(1+C^{\prime}(y)\right) C^{\prime \prime}(y)
$$

For a fixed value of $\phi$ there is therefore only a small window in $k$, namely when $\phi_{c}(k) \approx \phi$, for which $\partial_{t} Z(\phi)$ may differ significantly from zero. This means that, even for $\Lambda \rightarrow \infty$, the function $Z_{k}(\phi)$ changes between $k=\Lambda$ and $k=0$ by a finite amount at most. As another consequence, the anomalous dimension $\eta$ turns out very small for all $k$ except possibly in the window $k^{2} \approx \tilde{m}^{2}$. In fact, from

$$
\eta=-\frac{1}{Z(0)} \partial_{t} Z(0)=-\frac{\alpha^{2} \kappa^{2}}{2 \pi} \tilde{m}^{4} Z(0) \int_{0}^{\infty} d x x \tilde{\partial}_{t}\left\{\left(\frac{\partial P}{\partial x}\right)^{2}\left(P+\tilde{m}^{2} Z\right)^{-4}\right\}
$$

one infers easily $\eta \sim \alpha^{2} \tilde{m}^{4} / k^{4}$ for $k^{2} \gg \tilde{m}^{2}$ and $\eta \sim \alpha^{2} k^{2} / \tilde{m}^{2}$ for $k^{2} \ll \tilde{m}^{2}$. For the special case $C(y) \equiv 1$ one finds

$$
\partial_{t} Z=-\frac{\alpha^{2} \kappa^{2}}{3 \pi} \frac{\tilde{m}^{4} k^{2}}{\left(k^{2}+\tilde{m}^{2}\right)^{3}}
$$

where $Z \equiv Z_{k} \equiv Z_{k}(\phi=0)$.

\section{Flow of the Effective Liouville Action}

Next we solve the evolution equations derived in the previous sections. First we consider values of $\phi$ around zero and the limit $\tilde{m}^{2} \ll k^{2}$. In this limit the couplings 
$\kappa, \alpha \kappa, Z$ and $\zeta$ remain constant and we can use on the r.h.s. of the flow equations

$$
\kappa_{k}=\kappa_{\Lambda}, \alpha_{k} \kappa_{k}=\alpha_{\Lambda} \kappa_{\Lambda}, Z_{k}=\frac{\kappa_{\Lambda}^{2}}{4 \pi}, \zeta_{k}=1
$$

The only nontrivial running concerns the "mass parameter" $\tilde{m}_{k}^{2}=m_{k}^{2}$ which is governed by an anomalous mass dimension,

$$
\partial_{t} m^{2}=-4 \alpha_{\Lambda}^{2} m^{2}
$$

and similarly for $m_{i}^{2}$ :

$$
\partial_{t} m_{i}^{2}=-4 \alpha_{i \Lambda}^{2} m_{i}^{2}
$$

The solution

$$
m_{k}^{2}=\tilde{m}_{k}^{2}=\left(\frac{\Lambda}{k}\right)^{4 \alpha_{\Lambda}^{2}} m_{\Lambda}^{2} \quad, \quad m_{i k}^{2}=\left(\frac{\Lambda}{k}\right)^{4 \alpha_{i \Lambda}^{2}} m_{i \Lambda}^{2}
$$

leads to an increase of $m_{k}^{2}$ and of the ratio $\tilde{m}_{k}^{2} / k^{2}$ as $k$ is lowered. There is therefore necessarily a value of $k$ for which the approximation $\tilde{m}^{2} / k^{2} \ll 1$ breaks down. The ratio $\tilde{m}^{2} / k^{2}$ is the only relevant parameter appearing in the various threshold functions in the evolution equations. We conclude that for values $k^{2} \approx \tilde{m}_{k}^{2}$ there is a qualitative change in the behavior where the running of $\tilde{m}_{k}^{2}$ stops.

For a more detailed investigation of the "threshold behavior" at $\tilde{m}^{2} / k^{2} \approx$ 1 we start with the "wave function renormalization" $Z_{k}$. Since the anomalous dimension $\eta$ is effectively a function of $\tilde{m}^{2} / k^{2}$ which differs from zero only for $\tilde{m}^{2} / k^{2} \approx 1$ and vanishes both for $\tilde{m}^{2} / k^{2} \rightarrow 0$ and $\tilde{m}^{2} / k^{2} \rightarrow \infty$ there can be only a finite shift in $Z_{k}$ as $k$ crosses the threshold. Also $\alpha_{k} \kappa_{k}$ is effectively a function $\tilde{m}^{2} / k^{2}$,

$$
\frac{\alpha_{k} \kappa_{k}}{\alpha_{\Lambda} \kappa_{\Lambda}}=\mathcal{G}\left(\frac{\tilde{m}^{2}}{k^{2}}\right), \mathcal{G}(0)=1
$$

and the solution of (4.36) takes the general form

$$
\begin{aligned}
& Z_{k}=\frac{\kappa_{\Lambda}^{2}}{4 \pi}\left[1+\delta_{Z}\left(\frac{\tilde{m}^{2}}{k^{2}}\right)\right] \\
& \delta_{Z}(0)=0, \quad \delta_{Z}\left(\frac{\tilde{m}^{2}}{k^{2}} \rightarrow \infty\right)=\bar{\delta}
\end{aligned}
$$

for some constant $\bar{\delta}$. We can now insert (5.5) and (5.6) into the flow equation (4.27) for $\tilde{m}^{2}$ :

$$
\partial_{t} \tilde{m}^{2}=-4 \alpha_{\Lambda}^{2} \mathcal{K}\left(\frac{\tilde{m}^{2}}{k^{2}}\right) \tilde{m}^{2}
$$


The new threshold function $\mathcal{K}\left(\tilde{m}^{2} / k^{2}\right)$ obeys

$$
\begin{aligned}
& \mathcal{K}\left(\frac{\tilde{m}^{2}}{k^{2}}\right)=\frac{\mathcal{G}^{2}\left(\tilde{m}^{2} / k^{2}\right) \mathcal{J}\left(\tilde{m}^{2} / k^{2}\right)}{1+\delta_{Z}\left(\tilde{m}^{2} / k^{2}\right)}-\frac{\eta\left(\tilde{m}^{2} / k^{2}\right)}{4 \alpha_{\Lambda}^{2}} \\
& \mathcal{K}(0)=1, \quad \mathcal{K}\left(\frac{\tilde{m}^{2}}{k^{2}} \rightarrow \infty\right)=0
\end{aligned}
$$

The precise threshold behavior depends on the choice of the infrared cutoff function $C$ but the stopping of the flow of $\tilde{m}^{2}$ for $k^{2} \ll \tilde{m}^{2}$ is general.

The solution of the flow equation (4.9) for $\kappa$ can be written in the form

$$
\kappa_{k}^{2}=\kappa_{\Lambda}^{2}+\frac{1}{3} \int_{k^{2}}^{\Lambda^{2}} d k^{2} \frac{\alpha \kappa \tilde{m}^{2}}{\left(k^{2}+\tilde{m}^{2}\right)^{2}}
$$

The integral on the r.h.s. is convergent for $\Lambda \rightarrow \infty$ and leads to a finite shift in $\kappa$ as $k$ crosses the threshold at $k^{2} \approx \tilde{m}^{2}$. Setting $w=\tilde{m}^{2} / k^{2}$ and defining $h(w)$ by

$$
\frac{d h}{d k^{2}}=\frac{1}{k^{2}} \frac{\mathcal{G}(w) w}{(1+w)^{2}}
$$

one has

$$
\kappa_{k}^{2}=\kappa_{\Lambda}^{2}+\frac{\alpha_{\Lambda} \kappa_{\Lambda}}{3}\left[h\left(\frac{\tilde{m}_{\Lambda}^{2}}{\Lambda^{2}}\right)-h\left(\frac{\tilde{m}_{k}^{2}}{k^{2}}\right)\right]
$$

The 1.h.s. of the differential equation (5.10) for $h$ involves the threshold function $\mathcal{K}(w)$ of $(5.8)$ according to

$$
\begin{aligned}
\frac{d h}{d k^{2}} & =\frac{d h}{d w}\left(\frac{1}{k^{2}} \frac{\partial \tilde{m}^{2}}{\partial k^{2}}-\frac{\tilde{m}^{2}}{k^{4}}\right) \\
& =-\frac{d h}{d w}\left(2 \alpha_{\Lambda}^{2} \mathcal{K}(w) w+w\right) \frac{1}{k^{2}}
\end{aligned}
$$

Hence (5.10) is equivalent to

$$
\frac{d h}{d w}=-\frac{1}{\left(1+2 \alpha_{\Lambda}^{2}\right)(1+w)^{2}} \mathcal{H}(w)
$$

Here we have collected all details of the threshold functions in the factor

$$
\mathcal{H}(w) \equiv \frac{\left(1+2 \alpha_{\Lambda}^{2}\right) \mathcal{G}(w)}{1+2 \alpha_{\Lambda}^{2} \mathcal{K}(w)}
$$

If we neglect these details and approximate $\mathcal{H}(w)=1$ the integration of (5.13) is easily done and leads to

$$
\kappa_{k}^{2}=\kappa_{\Lambda}^{2}+\frac{1}{3} \frac{\alpha_{\Lambda} \kappa_{\Lambda}}{1+2 \alpha_{\Lambda}^{2}}\left[\frac{\tilde{m}_{k}^{2}}{k^{2}+\tilde{m}_{k}^{2}}-\frac{\tilde{m}_{\Lambda}^{2}}{\Lambda^{2}+\tilde{m}_{\Lambda}^{2}}\right]
$$


The second term in the bracket vanishes for $\Lambda \rightarrow \infty$. Also, we may use $\alpha_{\Lambda} \kappa_{\Lambda}=$ $1+2 \alpha_{\Lambda}^{2}$ from the Ward identity (3.30). We see that $\kappa_{k}^{2}$ increases by $1 / 3$ as $k$ moves through the threshold

$$
\kappa_{k}^{2}=\kappa_{\Lambda}^{2}+\frac{1}{3} \frac{\tilde{m}_{k}^{2}}{k^{2}+\tilde{m}_{k}^{2}}
$$

and, for $k=0$, we end up at

$$
\kappa_{0}^{2}=\kappa_{\Lambda}^{2}+\frac{1}{3}=\frac{26-c}{3}
$$

This is exactly the result we had hoped to find for a consistent truncation, namely that $3 \kappa_{k}^{2}$ moves from $25-c$ for $k=\Lambda$ to $26-c$ for $k=0$. A check if the details of the threshold functions do not spoil this picture is interesting but nontrivial. It requires a proof of the identity

$$
\int_{0}^{\infty} d w \frac{\mathcal{H}(w)}{(1+w)^{2}}=1
$$

As for the wave function renormalization, the Ward identities require that $\zeta_{k \rightarrow 0}=1$ which, according to eq. (5.6), amounts to

$$
\bar{\delta}=\frac{1}{25-c}=\frac{1}{3 \kappa_{\Lambda}^{2}}
$$

To check whether this is the case for our solution we combine eqs. (4.9) and (4.36) and find

$$
\frac{d \zeta}{d k^{2}}=\frac{\alpha^{2}}{3}\left[\frac{\zeta}{\alpha \kappa} \frac{\tilde{m}^{2}}{\left(k^{2}+\tilde{m}^{2}\right)^{2}}-\frac{2 \tilde{m}^{4}}{\left(k^{2}+\tilde{m}^{2}\right)^{3}}\right]
$$

Approximating the running of $\tilde{m}_{k}^{2}$ by eq. (5.4), neglecting the evolution of $\alpha_{k}$ and using $\zeta_{k} /\left(\alpha_{k} \kappa_{k}\right) \approx 1$ (note $(\alpha \kappa)_{k \rightarrow 0}=1$, see below) one obtains for $\Lambda \rightarrow \infty$

$$
\zeta_{k}=\zeta_{\Lambda}-\frac{\alpha_{\Lambda}}{3 \kappa_{\Lambda}}\left[\frac{\tilde{m}_{k}^{2}}{k^{2}+\tilde{m}_{k}^{2}}-\frac{\tilde{m}_{k}^{4}}{\left(k^{2}+\tilde{m}_{k}^{2}\right)^{2}}\right] \frac{\alpha_{k}^{2}}{\alpha_{\Lambda}^{2}}
$$

For $k \rightarrow 0$ the two terms in the bracket cancel and one indeed obtains in this approximation

$$
\zeta_{0}=\zeta_{\Lambda}=1
$$

We remark that the approximations leading to both $\mathcal{H}(w)=1$ and $\zeta_{0}=1$ become exact in the leading order of the semiclassical $(1 / c)$ expansion.

In order to understand the shape of the potential $U_{k}(\phi)$ for $k \rightarrow 0$ we have to reconcile the running of the prefactor of $\exp \left(2 \alpha_{\Lambda} \kappa_{\Lambda} \phi\right)$ for $\phi<\phi_{c}$ with the 
approximate $k$-independence of $U_{k}(\phi)$ for $\phi>\phi_{c}$. Expressed in different terms, we have to incorporate the threshold function in the running of the $\phi$-dependent mass term $\tilde{m}^{2} \exp (2 \alpha \kappa \phi)$ according to (4.28), (5.7). We will approximate here the threshold effects by a sharp threshold and make the approximation

$$
U_{k}(\phi)=\frac{Z_{\Lambda} \tilde{m}_{k}^{2}(\phi)}{4 \alpha_{\Lambda}^{2} \kappa_{\Lambda}^{2}} \exp \left(2 \alpha_{\Lambda} \kappa_{\Lambda} \phi\right)
$$

with

$$
\tilde{m}_{k}^{2}(\phi)=\left\{\begin{array}{cc}
\mu_{0} k^{-4 \alpha_{\Lambda}^{2}} & \text { for } \phi<\phi_{c}(k) \\
\mu_{0} k_{c}(\phi)^{-4 \alpha_{\Lambda}^{2}} & \text { for } \quad \phi>\phi_{c}(k)
\end{array}\right.
$$

and

$$
\mu_{0}=\tilde{m}_{\Lambda}^{2} \Lambda^{4 \alpha_{\Lambda}^{2}}=m_{\Lambda}^{2} \Lambda^{4 \alpha_{\Lambda}^{2}}
$$

Here $k_{c}(\phi)$ is the $\phi$-dependent scale where the running stops as $\phi_{c}(k)$ becomes smaller than $\phi$ for $k<k_{c}$. It is given by the relation

$$
\phi=\frac{1}{2 \alpha_{\Lambda} \kappa_{\Lambda}} \ln \frac{k_{c}^{2}}{\tilde{m}_{k}^{2}\left(k_{c}\right)}
$$

with $\tilde{m}_{k}^{2}\left(k_{c}\right)=\mu_{0} k_{c}^{-4 \alpha_{\Lambda}^{2}}$. This yields

$$
k_{c}(\phi)^{-4 \alpha_{\Lambda}^{2}}=\left[\mu_{0} \exp \left(2 \alpha_{\Lambda} \kappa_{\Lambda} \phi\right)\right]^{-\frac{2 \alpha_{\Lambda}^{2}}{1+2 \alpha_{\Lambda}^{2}}}
$$

In consequence, the potential for $\phi \gg \phi_{c}$ becomes independent of $k$ and approaches the limit

$$
U(\phi)=\frac{Z_{\Lambda}}{4 \alpha_{\Lambda}^{2} \kappa_{\Lambda}^{2}} \mu_{0}^{\frac{1}{1+2 \alpha_{\Lambda}^{2}}} \exp \left(\frac{2 \alpha_{\Lambda} \kappa_{\Lambda}}{1+2 \alpha_{\Lambda}^{2}} \phi\right)
$$

It is remarkable that the combination $\alpha_{\Lambda} \kappa_{\Lambda} /\left(1+2 \alpha_{\Lambda}^{2}\right)$ equals exactly unity by virtue of the Ward identity at $k=\Lambda$. One concludes that the effective exponent obeys

$$
\alpha_{k} \kappa_{k}=1 \quad \text { for } \quad k \ll k_{c}
$$

where $k_{c}=\tilde{m}$ if $\phi=0$. The effective potential for the quantum Liouville theory has the same exponential dependence as the classical potential,

$$
U(\phi)=\frac{\kappa_{0}^{2} \bar{m}^{2}}{16 \pi} \exp (2 \phi)
$$


but with a modified mass parameter

$$
\bar{m}^{2}=\left(m_{\Lambda}^{2} \Lambda^{4 \alpha_{\Lambda}^{2}}\right)^{\frac{1}{1+2 \alpha_{\Lambda}^{2}}}\left(\frac{\alpha_{0}}{\alpha_{\Lambda}}\right)^{2}
$$

Similarly, the term in the effective Lagrangian which is linear in the matter fields $\psi_{i}$ can be written in the form $\sqrt{g} \sum_{i} H_{i}(\phi) \psi_{i}$, with

$$
H_{i}(\phi)=\frac{\mu_{i} Z_{\Lambda}}{4 \alpha_{i \Lambda}^{2} \kappa_{\Lambda}^{2}} \exp \left(2 \alpha_{i \Lambda} \kappa_{\Lambda} \phi\right) \begin{cases}k^{-4 \alpha_{i \Lambda}^{2}} & \text { for } \phi<\phi_{c}(k) \\ k_{c}(\phi)^{-4 \alpha_{i \Lambda}^{2}} & \text { for } \phi>\phi_{c}(k)\end{cases}
$$

The reason is that if one expands (4.2) to first order in $\psi_{i}$ one finds that the running of $\tilde{m}_{i k}$, too, stops at $k \approx k_{c}$. (The threshold for $\tilde{m}_{i k}$ is determined by $\tilde{m}_{k}$, not by $\tilde{m}_{i k}$ itself.) With (5.23) this yields for $\phi \gg \phi_{c}(k)$

$$
H_{i}(\phi) \sim \exp \left[\left(2 \alpha_{i \Lambda} \kappa_{\Lambda}-4 \alpha_{i \Lambda}^{2}\right) \phi\right]
$$

or, with eq. (3.33),

$$
\lim _{k \rightarrow 0} \alpha_{i k} \kappa_{k}=\alpha_{i \Lambda} \kappa_{\Lambda}-2 \alpha_{i \Lambda}^{2}=1-\Delta_{i}^{0}
$$

In consequence, $\Delta_{i}(k)$ shifts away from $\Delta_{i}(\Lambda)$ as $k \rightarrow 0$, where it approaches again the "classical" value appearing in (2.23):

$$
\Delta_{i}(0)=1-\frac{\alpha_{i 0} \kappa_{0}}{\alpha_{0} \kappa_{0}}=\Delta_{i}^{0}
$$

In view of the KPZ relation this is certainly a surprising result and we will comment on it in the conclusions.

The actions $\Gamma_{k}^{L}$ and $\Gamma_{k}^{\psi}$ are completely determined now. In order to judge the quality of the truncation we can check how well $\Gamma_{k}^{L}+\Gamma_{k}^{\psi}$ satisfies the Weyl-Ward identities of section 3. (Recall that $\Gamma_{k}^{\text {grav }}$ was not included there.) As we mentioned already, the consistency of evolution equation and Ward identity is automatic for the exact solution, but it might be spoiled if we use a "bad" truncation. Let us insert the solutions for $m_{k}^{2}, \ldots$ into (3.13) and let us also use the conditions (3.30) and (3.33) which constrain the allowed initial conditions. We will concentrate on the two regions $\phi \ll \phi_{c}$ and $\phi \gg \phi_{c}$ and omit the more complicated threshold for $\phi$ near $\phi_{c}$. (We have not solved the evolution equation near the threshold with 
high precision either.) Above the threshold, for $\phi \ll \phi_{c}$ or large $k$, we find upon inserting our solution

$$
\begin{aligned}
& \int d^{2} x \sqrt{g} \mathcal{L}\left(\Gamma_{k}^{L}+\Gamma_{k}^{\psi}\right)+\frac{26-c+\tau}{24 \pi} \int d^{2} x \sqrt{g} R \\
& =-\frac{1}{4 \pi} m_{k}^{2}\left(\frac{\alpha_{\Lambda}}{\alpha_{k}}\right)^{2} \int d^{2} x \sqrt{g} e^{2 \alpha_{\Lambda} \kappa_{\Lambda} \phi} \\
& \quad-\frac{1}{4 \pi} \sum_{i} m_{i k}^{2}\left(\frac{\alpha_{i \Lambda}}{\alpha_{i k}}\right)^{2} \int d^{2} x \sqrt{g} \psi_{i} e^{2 \alpha_{i \Lambda} \kappa_{\Lambda} \phi} \\
& =\partial_{t}\left(\Gamma_{k}^{L}+\Gamma_{k}^{\psi}\right)
\end{aligned}
$$

This is precisely the exact global (integrated) Ward identity (2.30) with $\eta_{k}=0$. This is a very gratifying result which shows that our approximate renormalization group trajectory should not be too far away from the exact one in this region. The local Ward identity contains more information than the global one. For our truncation it is encoded in the equations (3.15) -(3.18). For $\tau=0$, eq. (3.16) with (3.22) yields

$$
3 \kappa^{2}=26-c-\frac{k^{2}}{k^{2}+\tilde{m}^{2}}
$$

On the other hand, since for large $k$ the threshold function $\mathcal{H}(w)$ equals unity, the solution of the flow equation is given by (5.16):

$$
3 \kappa^{2}=25-c+\frac{\tilde{m}^{2}}{k^{2}+\tilde{m}^{2}}
$$

This is exactly the same expression as (5.37). Similarly, for $\alpha_{k} \rightarrow \alpha_{\Lambda}, \kappa_{k} \rightarrow \kappa_{\Lambda}$ the result of the flow equation for $\zeta_{k}$, eq. (5.21), coincides with the Ward identity (3.15), (3.21). We also note that for large $k$ the running of $\zeta, \kappa \alpha \kappa$ and $\alpha_{i} \kappa$ can be neglected and the Ward identities (3.17), (3.18) are therefore obeyed.

Let us also turn to the Ward identities for $k=0$. Since $\mathcal{A}_{k}^{(n)}=0$, eqs. (3.15)-(3.18) imply

$$
\zeta_{0}=1, \quad 3 \kappa_{0}^{2}=26-c, \quad \alpha_{0} \kappa_{0}=1, \quad \alpha_{i 0} \kappa_{0}=1-\Delta_{i}^{0}
$$

These are exactly the values we have found from the solution of the flow equation. In particular, we note that the Ward identities (3.17), (3.18) imply $\Delta_{i}(0)=1-$ $\alpha_{i 0} \kappa_{0} / \alpha_{0} \kappa_{0}=\Delta_{i}^{(0)}$, a result that we have already found from the solution of the 
truncated evolution equation. We conclude that our truncation seems to work very well, both for large and for small $k$.

Before closing this section, we would like to comment on the use of a running $Z_{k}$ in the definition of the IR-cutoff $R_{k}(2.2)$. We have learned that $Z_{k}$ is only shifted by a finite amount once $k$ drops below the threshold value. In this particular case it can also be justified to use a constant $Z_{\Lambda}$ instead of a running $Z_{k}$ in the definition (2.2). In this version the last term $\sim \eta$ in the global Ward identity (2.30) is absent. Similarly, there is no second term $\sim B_{k}^{(\eta)}$ in the evolution equation (4.1). The price to pay is a somewhat more complicated momentum dependence of the propagator for $k$ below the threshold which is due to the finite shift of the coefficient of the $\left(D_{\mu} \phi\right)^{2}$ term. Since the running essentially stops in this region, this actually is not a very severe disadvantage.

\section{Liouville versus Feigin-Fuks Theory}

So far we have not yet determined the function $\tilde{\kappa}_{k}$ which appears in the pure gravity action (3.7). For $C \equiv 1$, say, its $\beta$-function is obtained by extracting the term proportional to the induced gravity action $I[g]$ from the r.h.s. of

$$
\partial_{t} \Gamma_{k}^{\text {grav }}[g]=\operatorname{Tr}\left[k^{2}\left(-D^{2}+k^{2}+E_{k}\right)^{-1}\right]
$$

We may set $\psi_{i}=0, \phi=0$ so that $E_{k}=\tilde{m}_{k}^{2}$. The induced gravity action $I[g]$ arises by integrating out massless fields. Massive fields would not give rise to structures as nonlocal as $R \Delta_{g}^{-1} R$. Therefore the r.h.s. of (6.1) does not contain a piece proportional to $I[g]$ and $\partial_{t} \tilde{\kappa}_{k}=0$. (This can be verified with the techniques of ref. [37].) Thus

$$
3 \widetilde{\kappa}_{k}^{2}=3 \tilde{\kappa}_{\Lambda}^{2}=26-c
$$

As a consequence, $\Gamma_{k}^{\text {grav }}$ contributes a piece $\mathcal{L} \Gamma_{k}^{\text {grav }}=(26-c) R /(24 \pi)$ to the conformal anomaly, and the global Ward identity for the complete action assumes the form

$$
\partial_{t} \Gamma_{k}-\int d^{2} x \sqrt{g} \mathcal{L} \Gamma_{k}=\frac{\tau}{24 \pi} \int d^{2} x \sqrt{g} R
$$


For the Weyl-invariant measure this equation is equivalent to the statement that $\Gamma_{k}$ is Weyl-invariant under the same transformations as the classical action, together with $k^{\prime}=k e^{-\sigma}$, whereby only $x^{\mu}$-independent $\sigma$ 's are allowed:

$$
\Gamma_{k^{\prime}}\left[\phi^{\prime} ; g_{\mu \nu}^{\prime}, \psi_{i}^{\prime}\right]=\Gamma_{k}\left[\phi ; g_{\mu \nu}, \psi_{i}\right]
$$

In (6.4) there is no anomaly term present. This is as it should be: When $k$ approaches zero, $\Gamma_{k}$ is heading for a conformal field theory of the combined ghost + matter + Liouville system whose total central charge is zero. Note also that $\tilde{\kappa}_{k}=\kappa_{k}$ and $\zeta_{k}=1$ is precisely what is needed to combine the $\left(D_{\mu} \phi\right)^{2}$ and $R \phi$-terms with the pure gravity term into a manifestly Weyl-invariant action $I\left[g_{\mu \nu} e^{2 \phi}\right]$.

In the above discussion we made the tacit assumption that $m_{\Lambda} \neq 0$. At this point a brief comparison of Liouville theory with $m_{\Lambda} \neq 0$ and the free field case $m_{\Lambda}=0$ is in order. If we start the evolution at a theory of the form

$$
\Gamma_{\Lambda}^{L}(m=0)=\frac{\kappa_{\Lambda}^{2}}{8 \pi} \int d^{2} x \sqrt{g}\left\{D_{\mu} \phi D^{\mu} \phi+R \phi\right\}
$$

then the Ward identities still tell us that $3 \kappa_{\Lambda}^{2}=25-c$ and $3 \tilde{\kappa}_{\Lambda}^{2}=26-c$. (We set $\tau=0$ and $\psi \equiv 0$ here.) The action (6.5) is a Feigin-Fuks [36] conformal field theory of a free field with a background charge whose nonminimal gravitational interaction shifts the central charge away from unity. Obviously (6.5) is invariant under the renormalization group evolution so that this time

$$
3 \kappa_{k=0}^{2}=3 \kappa_{\Lambda}^{2}=25-c \quad\left(m_{\Lambda}=0\right)
$$

For $m_{\Lambda}=0$ it is not true any more that the piece $\sim I[g]$ in $\Gamma_{k}^{\text {grav }}$ is a constant. The r.h.s. of (6.1) can be replaced by $\frac{1}{2} \partial_{t} \operatorname{Tr} \ln \left[-D^{2}+k^{2}\right]$ now, and this integrates to

$$
\Gamma_{k=0}^{\mathrm{grav}}[g]=\Gamma_{\Lambda}^{\mathrm{grav}}[g]+\frac{1}{2} \operatorname{Tr} \ln \left[-D^{2}\right]=-\frac{3 \tilde{\kappa}_{\Lambda}^{2}-1}{96 \pi} I[g]
$$

because $\operatorname{Tr} \ln \left[-D^{2}\right]=I[g] / 48 \pi$. Therefore

$$
3 \tilde{\kappa}_{k=0}^{2}=3 \tilde{\kappa}_{\Lambda}^{2}-1=25-c \quad\left(m_{\Lambda}=0\right)
$$

The combined theory $\Gamma_{0}^{L}+\Gamma_{0}^{\text {grav }}$ is conformal with total central charge zero. By eq. (2.20) the $\phi$-sector contributes $25-c$ and the gravity sector $-(25-c)$. This 
has to be contrasted with $m_{\Lambda} \neq 0$ where the net central charge zero arises form a compensation of $26-c$ in the $\phi$-sector and $-(26-c)$ in the pure gravity sector. Here the "pure gravity sector" is a substitute for the integrated-out matter and ghost system. In this sense we can confirm the usual statement that the $\phi$-field contributes to the central charge like an ordinary free boson, but we have also seen that depending on whether $m_{\Lambda}=0$ or $m_{\Lambda} \neq 0$ the mechanism of how this comes about is quite different.

\section{The UV-Fixed Point}

In this section we put Liouville field theory in a more general context by looking at the space of 2-dimensional theories with one scalar field as a whole. In general, a critical scalar theory is governed by a fixed point which typically is IR-unstable in one or several directions. The flow starts then for $k=\Lambda$ in the immediate vicinity of this fixed point and remains there for a long "running time". Depending on the value of $\phi$ the running stops at a certain point, and $\Gamma_{k}[\phi]$ becomes independent of $k$ for $k \rightarrow 0$. Generically there is also a region in $\phi$ where the running never stops. In the critical $\mathbf{Z}_{2}$-symmetric $\phi^{4}$-theory (Ising model) this region shrinks to one point - the potential minimum - as $k \rightarrow 0$. In our case this region is $\phi<\phi_{c}(k)$ and it moves to $\phi \rightarrow-\infty$ as $k \rightarrow 0$. We have argued in sect. 5 that the $k$-independent form of $\Gamma_{k \rightarrow 0}[\phi]$ is essentially the classical Liouville action. In this section we want to investigate closer the fixed point which governs the scaling behavior. In particular, we search for fixed points of the renormalization group flow for appropriate dimensionless variables. Though the fixed point equation is easier to solve than the evolution equation, it is impossible to find exact solutions unless one introduces approximations of some sort. In the following we truncate the space of actions by considering only functionals of the form ("local potential approximation")

$$
\Gamma_{k}[\phi ; g]=Z_{k} \int d^{2} x \sqrt{g}\left\{\frac{1}{2} D_{\mu} \phi D^{\mu} \phi+R f_{k}(\phi)+k^{2} u_{k}(\phi)\right\}
$$


Here $f_{k}$ and $u_{k}$ are arbitrary dimensionless functions. Their evolution is governed by (4.1),

$$
\begin{aligned}
& Z_{k} \int d^{2} x \sqrt{g}\left\{\partial_{t} u_{k}+2 u_{k}+\partial_{t} f_{k} k^{-2} R\right. \\
& \left.+Z_{k}^{-1} \partial_{t} Z_{k}\left[\frac{1}{2} k^{-2}\left(D_{\mu} \phi\right)^{2}+k^{-2} R f_{k}+u_{k}\right]\right\} \\
& =k^{-2} \operatorname{Tr}\left[\left\{\left(1-\frac{1}{2} \eta\right) C+\frac{D^{2}}{k^{2}} C^{\prime}\right\}\left[-\frac{D^{2}}{k^{2}}+C+\frac{R}{k^{2}} f_{k}^{\prime \prime}+u_{k}^{\prime \prime}\right]^{-1}\right]
\end{aligned}
$$

with $C \equiv C\left(-D^{2} / k^{2}\right)$, and $f_{k}^{\prime \prime}, u_{k}^{\prime \prime}$ denotes the second derivatives with respect to $\phi$. The interesting question is whether there exist fixed points $\left(u_{*}, f_{*}, Z_{*}\right)$ in the subspace of actions with the form (7.1). Because all parameters are chosen dimensionless, the condition is simply $\partial_{t} u_{k}=\partial_{t} f_{k}=\partial_{t} Z_{k}=0$. We emphasize that this is not the most general form of the fixed point even within the space of actions given by our truncation (7.1). In fact, for general scalar theories the anomalous dimension $\eta$ does not vanish at the fixed point. In this case one should replace $\phi$ by $k$-dependent renormalized fields $\phi_{R}=Z_{k}^{1 / 2} \phi$ in order to obtain the appropriate scaling solution. The condition $\partial_{t} Z_{k}=0$ selects therefore only the subclass of fixed point solutions with vanishing $\eta$. In view of the results of sects. 4 and 5 this is appropriate for "Liouville-type" critical scalar models.

For the determination of the fixed point potential $u_{*}(\phi)$ we may set $g_{\mu \nu}=\delta_{\mu \nu}$ and evaluate the trace in momentum space:

$$
8 \pi Z_{*} u_{*}(\phi)=\int_{0}^{\infty} d y \frac{C(y)-y C^{\prime}(y)}{y+C(y)+u_{*}^{\prime \prime}(\phi)}
$$

Note that by introducing $y \equiv p^{2} / k^{2}$ the equation has become independent of $k$, as it should. The r.h.s. of $(7.3)$ is not independent of $C$. For an exponential cutoff, say, the $y$-integral converges as it stands. For simplicity we use here $C \equiv 1$ and subtract from $u$ a constant which is not relevant for our purpose. With $\bar{u}(\phi)=u_{*}(\phi)-u_{*}\left(\phi_{0}\right)$ (where $\phi_{0}$ is arbitrary provided $u_{*}^{\prime \prime}\left(\phi_{0}\right)>-1$ ) eq. (7.3) can be written as

$$
\frac{d^{2}}{d \phi^{2}} \bar{u}(\phi)=-\frac{d \mathcal{P}}{d \bar{u}}(\bar{u}(\phi))
$$


with

$$
\mathcal{P}(\bar{u}) \equiv \bar{u}+p_{0} e^{-8 \pi Z_{*} \bar{u}}
$$

where $p_{0} \equiv\left(1+u_{*}^{\prime \prime}\left(\phi_{0}\right)\right) /\left(8 \pi Z_{*}\right)$. With the identification $\bar{u}(\phi) \hat{=} x(t)$ and $\mathcal{P}(\bar{u}) \hat{=} V(x)$, eq. (7.4) is Newton's equation for a particle located at $x(t)$ and moving in the potential $V(x)$. Because $\mathcal{P}(\bar{u})$ has a single minimum and $\mathcal{P}(\bar{u} \rightarrow \pm \infty) \rightarrow+\infty$, all solutions of (7.4) with an "energy"

$$
\mathcal{E} \equiv \frac{1}{2}\left(\frac{d \bar{u}}{d \phi}\right)^{2}+\mathcal{P}(\bar{u})
$$

larger than the minimum are oscillatory, i.e. $\bar{u}(\phi)$ is a periodic function? of $\phi$. Because the fixed points $\Gamma_{*}$ correspond to conformal field theories, one might have hoped to recover Liouville theory in this manner, but clearly an exponential $\bar{u}(\phi) \sim \exp (2 \phi)$ is not a solution of (7.4). In fact, the oscillatory solutions of (7.4) were discussed in ref. 27] already, where they were identified with the critical Sine-Gordon models. The only solution which is not oscillatory is the constant one, $\bar{u}(\phi)=\bar{u}_{\text {min }}$, where the "particle" sits for alle "times" $\phi$ at the minimum $\bar{u}_{\text {min }}$ of $\mathcal{P}$. It corresponds to a Gaussian (or more appropriately, Feigin-Fuks) fixed point for which the potential vanishes up to a constant. We are now going to show that Liouville theory can be understood as the perturbation of this fixed point by a relevant operator. When $k$ is lowered from infinity down to zero, this operator drives the theory from the IR unstable Gaussian fixed point to a different IR stable one which represents quantum Liouville theory and corresponds to our solution for $k=0$.

For an arbitrary cutoff function $C$ the constant fixed point solution reads

$$
u_{*}=\frac{1}{8 \pi Z_{*}} \int_{0}^{\infty} d y \frac{C(y)-y C^{\prime}(y)}{y+C(y)}
$$

It singles out a point in the space of all actions, and we are interested in the linearized flow in the tangent space at this very point. We write

$$
u_{k}(\phi)=u_{*}+\varepsilon \delta u_{k}(\phi)
$$

\footnotetext{
${ }^{9}$ This is also true for an arbitrary cutoff function $C$.
} 
with $(t \equiv \ln (k / \Lambda))$

$$
\delta u_{k}(\phi)=e^{-\gamma t} \Upsilon(\phi)+\sum_{i} e^{-\gamma_{i} t} e^{2 \Delta_{i}^{0} t} \psi_{i} \Upsilon_{i}(\phi)
$$

and expand the evolution equation to first order in $\varepsilon$. Here $\Upsilon(\phi)$ and $\Upsilon_{i}(\phi)$ are the eigenvectors of the linearized evolution operator with the eigenvalues $\gamma$ and $\gamma_{i}$, respectively. They are obtained explicitly by inserting (7.8) with (7.9) into (7.2) with $g_{\mu \nu}=\delta_{\mu \nu}$. The terms independent of $\varepsilon$ cancel by virtue of (7.7), and the terms linear in $\varepsilon$ involve a momentum space integral which is precisely of the type (4.21). It is independent of the form of $C$. Thus one finds

$$
\begin{gathered}
\Upsilon^{\prime \prime}(\phi)+4 \pi Z_{*}(2-\gamma) \Upsilon(\phi)=0 \\
\Upsilon_{i}^{\prime \prime}(\phi)+4 \pi Z_{*}\left(2-\gamma_{i}+2 \Delta_{i}^{0}\right) \Upsilon_{i}(\phi)=0
\end{gathered}
$$

At this point we understand what is so special about the exponential interaction potentials: They are the eigenvectors of the linearized renormalization group flow at the Gaussian fixed point. Parametrizing the solutions as $\Upsilon \sim \exp \left(2 \alpha_{*} \kappa_{*} \phi\right)$ and $\Upsilon_{i} \sim \exp \left(2 \alpha_{i *} \kappa_{*} \phi\right)$, and using $4 \pi Z_{*}=\kappa_{*}^{2}$ we obtain

$$
\begin{gathered}
\gamma=2+4 \alpha_{*}^{2} \\
\gamma_{i}=2+4 \alpha_{i *}^{2}+2 \Delta_{i}^{0}
\end{gathered}
$$

Hence, near the fixed point, the potential scales according to

$$
U_{k}(\phi)=\varepsilon Z_{*} k^{2} \delta u_{k}(\phi)=\frac{m_{\Lambda}^{2}}{16 \pi \alpha_{*}^{2}}\left[\left(\frac{\Lambda}{k}\right)^{4 \alpha_{*}^{2}} e^{2 \alpha_{*} \kappa_{*} \phi}+\sum_{i} \psi_{i}\left(\frac{\Lambda}{k}\right)^{4 \alpha_{i *}^{2}} e^{2 \alpha_{i *} \kappa_{*} \phi}\right]
$$

where we have adapted the free multiplicative integration constant to the notation (4.16). Obviously, for $\alpha_{*}, \alpha_{i *}$ real, the perturbation grows for decreasing $k$ and the fixed point is IR-unstable. If we identify $\alpha_{*}, \alpha_{i *}$ and $\kappa_{*}^{2}$ with the UV values $\alpha_{\Lambda}, \alpha_{i \Lambda}$ and $\kappa_{\Lambda}$, then eq. (7.14) coincides precisely with the solution of the evolution equation in section 5. This shows that the truncation employed there is rather accurate for large values of $k$ when the flow is still well approximated by its linearization around the Gaussian fixed point. 
We have not determined the function $f_{*}(\phi)$ yet. Because at the fixed point the 1.h.s. of (7.2) contains no $\sqrt{g} R f_{k}(\phi)$ term, the trace on the r.h.s. must not generate such a term. A sufficient condition for this not to happen is that $f_{*}(\phi)=c_{1}+c_{2} \phi$. Imposing in addition that the fixed point action $\Gamma_{*}$ satisfies the Ward identity we see that $\Gamma_{*}$ is precisely the Feigin-Fuks-type theory (6.5). The coefficient $\kappa_{*}^{2}$ is not determined by the fixed point condition, but the Ward identity requires it to be $3 \kappa_{*}^{2}=3 \kappa_{\Lambda}^{2}=25-c+\tau$.

To conclude, let us summarize the general picture as it arises form our investigation of the Ward identities, the evolution equation and the fixed point equation. The renormalization group trajectory starts at $k=\Lambda$ at a conformal theory with central charge $3 \kappa_{\Lambda}^{2}=25-c$ (for the Weyl invariant measure). This initial point approaches for $\Lambda \rightarrow \infty$ a Feigin-Fuks free field action $\Gamma_{\Lambda}^{L}(m=0)$ as in eq. (6.5). The relevant perturbations at this fixed point are governed by eigenvectors of the linearized flow, $\Upsilon(\phi)$ and $\Upsilon_{i}(\phi)$, which have an exponential dependence on $\phi$. Adding such a perturbation to $\Gamma_{\Lambda}^{L}(m=0)$ we obtain the Liouville action. The perturbation drives the system away from the IR-unstable fixed point. For $k \rightarrow 0, \Gamma_{k}^{L}$ approaches an IR-stable fixed point, a conformal theory with central charge $26-c$. 四 The exact Weyl Ward identity guarantees that $\Gamma_{k \rightarrow 0}^{L}$ is conformal with $c\left[\Gamma_{k \rightarrow 0}\right]=26-c$. With our truncation we find that $\Gamma_{0}^{L}$ equals the classical Liouville action. We expect that this is a very good approximation (and perhaps even exact) for the low momentum part of the effective action.

In this context it is important to keep in mind that the area operator is required to be an $(1,1)$ operator in the quantum theory, i.e., for $k \rightarrow 0$, but not at $k=\Lambda$. In the UV it is not marginal and this is what drives the system away from the UV-fixed point. In fact, to a conformal reparametrization $z \rightarrow z^{\prime}(z)$, $d s^{\prime 2}=\left|d z^{\prime} / d z\right|^{2} d s^{2}, \phi^{\prime}=\phi-\frac{1}{2} \ln \left|d z^{\prime} / d z\right|^{2}$ the area operator responds according to

$$
\left(\sqrt{g} e^{2 \alpha_{\Lambda} \kappa_{\Lambda} \phi}\right)^{\prime}=\left|\frac{d z^{\prime}}{d z}\right|^{-4 \alpha_{\Lambda}^{2}} \sqrt{g} e^{2 \alpha_{\Lambda} \kappa_{\Lambda} \phi}
$$

with the familiar exponent $4 \alpha_{\Lambda}^{2}$. This is also reflected by the presence of the field-dependent terms in $T_{\mu}^{\mu}\left[\Gamma_{\Lambda}^{L}\right]$ which we found in section 3 .

\footnotetext{
${ }^{10}$ This is not in contradiction with the Zamolodchikov $c$-theorem because $k$ is an IR cutoff.
} 
It is interesting that recently evidence for a similar mechanism in $d>2$ was found [38], 39]. Halpern and Huang investigated the linearized renormalization group flow at the Gaussian fixed point in $\mathrm{O}(\mathrm{N})$ symmetric scalar theories. They find non-polynomial interactions for which the Gaussian fixed point is IR-unstable rather than IR attractive (as in the case of polynomial potentials). Hence these theories are asymptotically free rather than trivial. It is tempting to regard them as higher dimensional counterparts of Liouville theory. In fact, for $d>2$ the potentials behave as $U(\phi) \sim \exp \left[a(d-2) \phi^{2}\right]$ for large $\phi$, where $a$ is a constant. These results have been questioned in ref. 40, however.

\section{Conclusion}

In this paper we have developed the general framework for the quantization of Liouville theory using exact renormalization group equations. We applied this program to a specific truncation of the space of actions. An important role was played by the Ward identities resulting from the Weyl invariance of the theory. They imply that the exact renormalization group trajectory $\Gamma_{k}$ runs in the IR into a fixed point which is a conformal field theory of central charge $26-c$. For $k \rightarrow \infty$ the Ward identities give rise to constraints on the initial values of the parameters contained in $\Gamma_{\infty}$. Only if these constraints are satisfied, the evolution drives $\Gamma_{k}$ towards the correct IR fixed point. Other initial values would correspond to other universality classes. In particular, the initial values for $\alpha_{\Lambda}$ and $\alpha_{i \Lambda}$ are fixed in terms of $c$ and the bare scaling dimensions $\Delta_{i}^{0}$ by eqs. (3.31) and (3.35). These formulas are well known from the standard approaches where they arise from the requirement that the area operator and the gravitationally dressed matter operator have dimensions $(1,1)$ in the quantum theory so that they can be integrated over the Riemann surface. In our case the interpretation is different: A priori these relations are statements about the microscopic or classical theories $\Gamma_{\infty}$ which are within the "range of attraction" of the correct IR fixed point, i.e., the evolution (quantization) is still to be performed. In section 4 we obtained the solution of the truncated evolution equation, and we saw that the 
gravitationally modified dimensions $\Delta_{i}(k)$ are renormalization group invariants only for large enough $k$. Only in this range the KPZ formula is reproduced. For $k \rightarrow 0$ the dimensions $\Delta_{i}(k)$ shift back to the "bare" values $\Delta_{i}^{0}$, which apply without quantized gravity.

As for our solution of the evolution equation, it is quite impressive to see the high degree of "conspiracy" among the $\beta$ functions of $\alpha_{k}, \kappa_{k}, \tilde{\kappa}_{k}$ and $m_{k}$. When $k$ is lowered, $m_{k}$ grows precisely at a rate which cuts off the running of $\kappa_{k}$ in such a manner that it changes during an infinitely long running time by a finite amount only. Though the Liouville "mass" is crucial for this mechanism, the same shift obtains also for $m_{\Lambda}=0$. The $\beta$-functions of $\kappa_{k}$ and $\tilde{\kappa}_{k}$ conspire in such a way that for $m_{\Lambda}=0$ the task of changing the central charge can be transferred from $\kappa_{k}$ to $\tilde{\kappa}_{k}$.

In this paper we used a truncation which is inspired by the classical Liouville action. It contains the same types of operators but with $k$-dependent coefficients. In the case of standard (scalar) theories truncations of this type were already successfully used in a variety of applications [23], [27], [21]. We have checked the quality of the truncation by comparing the solution of the evolution equation to the constraints imposed by the Ward identities. It turned out to be very satisfactory both for $k \rightarrow \infty$ and $k \rightarrow 0$. We believe that our truncation indeed reflects the most important qualitative and quantitative features of the quantum Liouville theory in the range considered, i.e., for $c \leq 1$ and for low momenta.

By combining the informations about the renormalization-group trajectory obtained from the Ward identities, the evolution equation and the fixed-point equation we are led to consider Liouville field theory for $c<1$ as a crossover phenomenon from one renormalization group fixed point to another. The trajectory starts at an UV-stable Feigin-Fuks ("Gaussian") fixed point. The area operator is a relevant operator there and it drives the trajectory towards a second, IR-stable fixed point, the quantum Liouville theory with central charge $26-c$. In our truncation the effective action of the latter equals the classical Liouville action with a renormalized parameter $m^{2}$.

It remains an important open question how to improve the truncation for 
$k \rightarrow 0$, and how to recover the KPZ scaling laws, for instance. It is quite clear that finding such an improved truncation will be very difficult because calculating arbitrary correlators in Liouville theory is known to be a formidable task, and these correlators contain the same information as $\Gamma_{k \rightarrow 0}$. If one naively adds further terms to the ansatz for $\Gamma_{k}$ one sees immediately that it is very hard to produce to "good" truncation which does not lead to inconsistencies with the Ward identities.

It should be emphasized that the present work deals essentially with the low momentum behavior of quantum Liouville theory. This is manifest in the study of the effective potential which generates the $n$-point functions at zero momentum. Also the investigation of the kinetic term was restricted to the lowest order in a derivative expansion and therefore to the limit of the lowest order of a Taylor expansion around vanishing momentum. It is a very interesting question what happens to $n$-point functions with typical external (euclidean) momentum squared $q^{2}>0$. The main observation here is that, for generic external momenta, $q^{2}$ acts as an effective infrared cutoff, very similar to $k^{2}$. We therefore suggest that for $q^{2}>0$ the $n$-point functions of quantum Liouville theory $(k=0)$ are qualitatively described by the generating functional $\Gamma_{k}$ evaluated at zero momentum, and with $k^{2}$ chosen such that $k^{2} \approx q^{2}$. In other words, the role of the effective infrared cutoffs $k^{2}$ and $q^{2}$ can be interchanged. If this simple picture holds true, our study of the effective action for arbitrary $k$ provides important information on the momentum dependence of the $n$-point functions of quantum Liouville theory. In particular, we expect a different behavior for $q^{2}$ larger or smaller than $q_{c}^{2} \equiv \bar{m}^{2} \exp (2 \phi)$. (Note the $\phi$-dependence of the critical momentum $q_{c}^{2}$, which is necessary for a conformal theory.) For $q^{2}<q_{c}^{2}$ the $n$-point functions are essentially given by $\Gamma_{k \rightarrow 0}$ as discussed in this work, i.e., the classical Liouville action. On the other hand, large momenta $q^{2}>q_{c}^{2}$ should be described by the $k \rightarrow \infty$ limit of $\Gamma_{k}$. It is in this region of large momenta that the operators $\psi_{i}$ scale according to the KPZ rule. This observation leads naturally to the idea of effective momentumdependent dimensions $\Delta_{i}\left(q^{2}\right)$, in close analogy to $\Delta_{i}(k)$. Furthermore, one expects a shift in the effective value of $\kappa$ and $\alpha \kappa$ as $q^{2}$ crosses the threshold $q_{c}^{2}$. Finally the factor $k^{-4 \alpha_{\Lambda}^{2}}$ in front of the exponential potential should now be translated into an 
effective momentum-dependent form factor which behaves for generic momenta as $\left(q^{2}\right)^{-2 \alpha_{\Lambda}^{2}}$. The precise behavior near the threshold $q_{c}^{2}$ is difficult, but in order to get an idea of the qualitative behavior we can approximate the threshold effects by a form factor $\left(\frac{q^{2}+q_{c}^{2}}{q_{c}^{2}}\right)^{-2 \alpha_{\Lambda}^{2}}$. As an example, this would lead to a characteristic behavior of the inverse propagator as

$$
q^{2}+\left[\bar{m}^{2} \exp \left(2 \beta\left(q^{2}\right) \phi\right)\right]^{1+2 \alpha_{\Lambda}^{2}}\left[q^{2}+\bar{m}^{2} \exp \left(2 \beta\left(q^{2}\right) \phi\right)\right]^{-2 \alpha_{\Lambda}^{2}}
$$

with $\beta\left(q^{2} \gg q_{c}^{2}\right)=\alpha_{\Lambda} \kappa_{\Lambda}$ and $\beta\left(q^{2} \ll q_{c}^{2}\right)=1$. In particular, this propagator becomes a massless propagator for $q^{2} \rightarrow \infty$. All this would have important implications for the general picture of $n$-point functions in quantum Liouville theory and certainly merits further study.

In most of the discussion we had to restrict ourselves to $c \leq 1$. In our approach the " $c=1$ barrier" is encountered if one tries to find an admissible initial point $\Gamma_{\infty}$ within the truncated space of actions. As a consequence of the Ward identity, $\alpha_{\Lambda} \kappa_{\Lambda}$ is complex for $1<c<25$ and such a point simply does not exist. Hence, if it exists at all, $\Gamma_{\infty}$ should be of a form rather different from the Liouville action. Here we are in the somewhat unusual situation that the quantization of a theory has very little to do with its classical action: $S_{L}$ enters only via its symmetry properties in the derivation of the Ward identity. Of course, by the very idea of universality, this is not a problem of principle but rather the generic situation: Universality classes are fixed essentially by the field content and the symmetries of a theory. The precise form of the initial action does not matter as long as it is attracted towards the correct IR fixed point. However, from a purely practical point of view it is quite disturbing that the classical action is of so little help in finding a representative of the desired universality class. It is one of the advantages of our formulation that the technically simpler step of finding $\Gamma_{\infty}$ is decoupled from the problem of the actual quantization (evolution). An investigation of the region $1<c<25$ has to start with an examination of the Ward identity (2.25) for a truncation as general as computationally feasible. The technically difficult part is the evaluation of $\mathcal{A}_{k}, k \rightarrow \infty$, for the assumed form of $\Gamma_{k}$, see eq. (2.27). If rather complicated nonlocal functions of $-D^{2}$ become important, $\Gamma_{k}^{(2)}$ is a complicated nonminimal differential operator in general, and 
almost nothing is known about the heat-kernel expansion of $\Gamma_{k}^{(2)}$ in a fairly general form. Thus any progress in this direction depends crucially on our ability to handle the Seeley-DeWitt expansion of such nonminimal operators. (For $k \rightarrow \infty$ it is sufficient to know the first few terms in this expansion in order to calculate $\mathcal{A}_{k}(x)$.)

In conclusion we think that the investigation of exact evolution equations is a promising new approach which puts Liouville theory into a novel perspective and provides new calculational tools which have not been exploited so far.

Acknowledgement: M. R. would like to thank G. Weigt for the numerous discussions which triggered these investigations. He is also grateful to A. Bonanno, M. Bonini, H. Dorn, H. Nicolai, J. Teschner, N. Tetradis and A. Wipf for helpful conversations. 


\section{References}

[1] J. Liouville, J. Math. Pures Appl. 18 (1853) 71

[2] T. L. Curtright, C. B. Thorn, Phys. Rev. Lett. 48 (1982) 1309;

E. Braaten, T. Curtright, C. B. Thorn, Phys. Lett. B118 (1982) 115;

Ann. Phys. 147 (1983) 365

[3] J. L. Gervais, A. Neveu, Nucl. Phys. B224 (1983) 329

[4] H. J. Otto, G. Weigt, Phys. Lett. B159 (1985) 341; Z. Phys. C31 (1986) 219

G. Weigt, Phys. Lett. B277 (1992) 79

[5] Y. Kazama, H. Nicolai, Int. J. Mod. Phys. A9 (1994) 667

[6] E. D'Hoker, R. Jackiw, Phys. Rev. D26 (1982) 3517

[7] M. Goulian, M. Li, Phys. Rev. Lett. 66 (1991) 2051

[8] J. L. Gervais, Commun. Math. Phys. 130 (1990) 257; 138 (1991) 301;

Nucl. Phys. B391 (1993) 287;

E. Cremmer, J. L. Gervais, J. F. Roussel, Commun. Math. Phys. 161 (1994)

597; Preprints hep-th/9506040, hep-th/9601034

[9] J. L. Gervais, J. Schnittger, Nucl. Phys. B413 (1994) 433;

J. Schnittger, Preprint hep-th/9512125;

J. Teschner, Preprint DESY 95-118 (1995)

[10] R. Dijkgraaf, E. Witten, Nucl. Phys B342 (1990) 486

J. Distler, Nucl. Phys. B342 (1990) 523;

E. Verlinde, H. Verlinde, Nucl. Phys. B348 (1990) 457

[11] A. M. Polyakov, Phys. Lett. B103 (1981) 207, 211

[12] J. Polchinski, Nucl. Phys. B324 (1989) 123 
[13] A. M. Polyakov, Mod. Phys. Lett. A2 (1987) 893;

V. Krizhnik, A. M. Polyakov, A. Zamolodchikov, Mod. Phys. Lett. A3 (1988) 819

[14] A. Chamseddine, M. Reuter, Nucl. Phys. B317 (1989) 757

[15] E. Abdalla, M. C. B. Abdalla, D. Dalmazi, A. Zadra, "2D-Gravity in Non-Critical Strings", Springer, New York, 1994

[16] K. G. Wilson, Phys. Rev. B4 (1971) 3174, 3184;

K. G. Wilson, J. Kogut, Phys. Rep. 12 (1974) 75;

F. Wegner, A. Houghton, Phys. Rev. A8 (1973) 401;

S. Weinberg, "Critical Phenomena for Field Theorists",

Erice Subnucl. Phys. (1976) 1;

J. Polchinski, Nucl. Phys. B231 (1984) 269

[17] A. Hasenfratz, P. Hasenfratz, Nucl. Phys. B270 (1986) 685;

B. Warr, Ann. Phys. 183 (1988) 1, 59;

T. Hurd, Commun. Math. Phys. 124 (1989) 153;

G. Keller, C. Kopper, Phys. Lett. B273 (1991) 323;

U. Ellwanger, Z. Phys. C58 (1993) 619;

U. Ellwanger, L. Vergara, Nucl. Phys. B398 (1993) 52;

M. Bonini, M. D'Attanasio, G. Marchesini, Nucl. Phys. B409 (1993) 441, B418 (1994) 81, B437 (1995) 163;

M. Alford, Phys. Lett. B336 (1994) 237;

M. Alford, J. March-Russell, Nucl. Phys. B417 (1994) 527;

S.-B. Liao, J. Polonyi, D. Xu, Phys. Rev. D51 (1995) 748;

P. E. Haagensen, Y. Kubyshin, J. I. Latorre, E. Moreno,

Phys. Lett. B323 (1994) 330;

A. Bonanno, Phys. Rev. D52 (1995) 969;

J. Comellas, Y. Kubyshin, E. Moreno, Preprint hep-th/9601112;

U. Kerres, G. Mack. G. Palma, Preprint hep-lat/9505008;

M. Griessl, G. Mack, Y. Xylander, G. Palma, Preprint hep-lat/9602014 
[18] C. Wetterich, Nucl. Phys. B352 (1991) 529, Z. Phys. C57 (1993) 451

[19] M. Reuter, C. Wetterich, Nucl. Phys. B391 (1993) 147; B408 (1993) 91

[20] C. Wetterich, Phys. Lett. B301 (1993) 90

[21] M. Reuter, C. Wetterich, Nucl. Phys. B417 (1994) 181; B427 (1994) 291

[22] M. Reuter, Preprint DESY 96-065 and hep-th/9604124

[23] N. Tetradis, C. Wetterich, Nucl. Phys. B398 (1993) 659, Int. J. Mod. Phys. A9 (1994) 4029, Nucl. Phys. B422 (1994) 541;

M. Gräter and C. Wetterich, Phys. Rev. Lett. 75 (1995) 378;

N. Tetradis, D. Litim, Preprint hep-th/9512073; Preprint hep-th/9501042;

B. Bergerhoff, F. Freire, D. Litim, S. Lola, C. Wetterich, Preprint hep-ph/950334, to appear in Phys. Rev. B

[24] M. Reuter, Phys. Rev. D53 (1996) 4430 and hep-th/9511128

[25] M. Reuter, Nonperturbative Evolution Equation for Quantum Gravity, Preprint DESY 96-080 and hep-th/9605030

[26] M. Reuter in: Proceedings of the 5th Hellenic School and Workshops on Particle Physics, Corfu, Greece, 1995; hep-th/9602012

[27] T. R. Morris, Phys. Lett. B345 (1995) 139, B329 (1994) 241, B334 (1994) 355

[28] U. Ellwanger, Phys. Lett. B335 (1994) 364;

U. Ellwanger, M. Hirsch, A. Weber, Preprint hep-th/9506019

[29] C. Wetterich, Z. Phys. C60 (1993) 461

[30] J. Distler, H. Kawai, Nucl. Phys. B321 (1989) 509

[31] N. Mavromatos, J. Miramontes, Mod. Phys. Lett. A4 (1989) 1849

[32] E. D'Hoker, P. S. Kurzepa, Mod. Phys. Lett. A4 (1990) 1411; E. D'Hoker, Mod. Phys. Lett. A6 (1991) 745 
[33] E. D'Hoker, Lectures at the VI Swieca Summer School, 1991, UCLA/91/TEP/35;

P. Ginsparg, G. Moore, Lectures at the 1992 TASI summer school, hep-th/9304011

[34] F. David, E. Guitter, Euro. Phys. Lett. 3 (1987) 1169;

F. David, Mod. Phys. Lett. A3 (1988) 1651

[35] H. Dorn, H.-J. Otto, Phys. Lett. B280 (1992) 204; Preprint hep-th/9501019;

H. Dorn, Phys. Lett. B343 (1995) 81; Preprint hep-th/9512023

[36] A. Chodos, C. Thorn, Nucl. Phys. B72 (1974) 509;

V. S. Dotsenko, V. A. Fateev, Nucl. Phys. B240 [FS12] (1984) 312,

B251 [FS13] (1985) 691

[37] A. O. Barvinsky, G. Vilkovisky, Nucl. Phys. B282 (1987) 163;

B333 (1990) 471, 512

[38] K. Halpern, K. Huang, Phys. Rev. Lett. 74 (1995) 3526;

Preprint hep-th/9510240

[39] V. Perival, Preprint hep-th/9512108

[40] T. R. Morris, Preprint hep-th/9601128 